\title{
Functional Clusters of Neurons in Layer 6 of Macaque V1
}

\author{
Michael J. Hawken, Robert M. Shapley, Anita A. Disney, Virginia Garcia-Marin, Andrew Henrie, Christopher A. Henry, \\ Elizabeth N. Johnson, Siddhartha Joshi, -Jenna G. Kelly, @Dario L. Ringach, and @Dajun Xing \\ Center for Neural Science, New York University, New York, New York 10003
}

Layer 6 appears to perform a very important role in the function of macaque primary visual cortex, V1, but not enough is understood about the functional characteristics of neurons in the layer 6 population. It is unclear to what extent the population is homogeneous with respect to their visual properties or if one can identify distinct subpopulations. Here we performed a cluster analysis based on measurements of the responses of single neurons in layer 6 of primary visual cortex in male macaque monkeys (Macaca fascicularis) to achromatic grating stimuli that varied in orientation, direction of motion, spatial and temporal frequency, and contrast. The visual stimuli were presented in a stimulus window that was also varied in size. Using the responses to parametric variation in these stimulus variables, we extracted a number of tuning response measures and used them in the cluster analysis. Six main clusters emerged along with some smaller clusters. Additionally, we asked whether parameter distributions from each of the clusters were statistically different. There were clear separations of parameters between some of the clusters, particularly for f1/f0 ratio, direction selectivity, and temporal frequency bandwidth, but other dimensions also showed differences between clusters. Our data suggest that in layer 6 there are multiple parallel circuits that provide information about different aspects of the visual stimulus.

Key words: layer 6; primate; visual cortex

\section{Significance Statement}

The cortex is multilayered and is involved in many high-level computations. In the current study, we have asked whether there are subpopulations of neurons, clusters, in layer 6 of cortex with different functional tuning properties that provide information about different aspects of the visual image. We identified six major functional clusters within layer 6 . These findings show that there is much more complexity to the circuits in cortex than previously demonstrated and open up a new avenue for experimental investigation within layers of other cortical areas and for the elaboration of models of circuit function that incorporate many parallel pathways with different functional roles.

\section{Introduction}

The study was motivated by the growing realization that layer 6 has multiple functional roles in the visual function of macaque

Received June 14, 2019; revised Jan. 26, 2020; accepted Jan. 27, 2020.

Author contributions: M.J.H., R.M.S., A.A.D., A.H., C.A.H., E.N.J., S.J., D.L.R., and D.X. designed research; M.J.H., R.M.S., A.A.D., A.H., C.A.H., E.N.J., S.J., D.L.R., and D.X. performed research; M.J.H., R.M.S., A.A.D., A.H., C.A.H., V.G.-M., E.N.J., S.J., J.G.K., D.L.R., and D.X. analyzed data; M.J.H., R.M.S., A.A.D., A.H., C.A.H., V.G.-M., E.N.J., S.J., J.G.K., D.L.R., and D.X. wrote the paper.

This work was supported by National Institutes of Health Grants EY1472, EY8300, and EY15549 to R.M.S. and M.J.H., Core Grant P30 EY13079, and Training Grant T32 EY7136. D.X. was supported by the Fundamental Research Funds for the Central Universities, 111 Project Grant BP0719032, and National Natural Science Foundation of China Grant 31371110 .

The authors declare no competing financial interests.

Correspondence should be addressed to Michael J. Hawken at michael.hawken@nyu.edu.

A.A. Disney's present address: Department of Neurobiology, Duke University, Durham, North Carolina 27110.

V. Garcia-Marin's present address: Department of Biology, York College, City University of New York, Jamaica, New York 11451.

C.A. Henry's present address: Department of Neuroscience, Albert Einstein College of Medicine, Bronx, New York 10461.

E.N. Johnson's present address: Wharton Neuroscience Initiative, University of Pennsylvania, Philadelphia, PA 19104.

S. Joshi's present address: Department of Neuroscience, University of Pennsylvania, Philadelphia, PA 19104. primary visual cortex, V1. Layer 6 provides major output from V1 to extrastriate (Fries et al., 1985) and subcortical targets (Fitzpatrick et al., 1994) that have different functional characteristics. Layer 6 also has multiple distinct intracortical recurrent pathways and provides long-range connections within the infragranular layers of V1. Layer 6 feedback afferents (McGuire et al., 1984; Wiser and Callaway, 1996) provide a considerable fraction of the excitatory synapses in V1 layer 4C. Furthermore, such layer 6 feedback to layer $4 \mathrm{C}$ is provided by distinct subpopulations (Wiser and Callaway, 1996) and appears to be of considerable importance in the generation of the dynamical responses within layer 4C $\alpha$ (Chariker et al., 2016, 2018).

The goal of the current study was to examine layer 6 neuron responses along multiple stimulus dimensions to seek functional

D.L. Ringach's present address: Department of Neurobiology, David Geffen School of Medicine at UCLA, Los Angeles, CA 90095

D. Xing's present address: Key State Laboratory of Cognitive Neuroscience and Learning, Beijing Normal University, Beijing 100875, People's Republic of China.

https://doi.org/10.1523/JNEUROSCI.1394-19.2020

Copyright $\odot 2020$ the authors 
clusters of layer 6 neurons by means of hierarchical clustering analysis. As shown in Results, distinct functional clusters emerged in the analysis. After presenting the functional clusters, we discuss their possible functional roles and the relation of functional clusters to classes of layer 6 neurons with different anatomical circuit motifs (Wiser and Callaway, 1996; Briggs and Callaway, 2001).

Although layer 6 in the primate has a number of distinct morphological neuronal populations (Callaway, 1998; Briggs et al., 2016), there have been few comprehensive studies of the visual physiology of layer 6 neurons in primate. However, there are indications of functional subpopulations in the anatomical data. Most layer 6 excitatory neurons have intracortical axonal projections (Lund and Boothe, 1975; Fitzpatrick et al., 1985; Wiser and Callaway, 1996), and some of those intracortically projecting neurons have M- or P-pathway-specific axonal projections, targeting either layer $4 \mathrm{C} \alpha$ or $4 \mathrm{C} \beta$ and $4 \mathrm{~A}$. There are other layer 6 subpopulations that project specifically to extrastriate areas, including MT (Lund et al., 1975; Fries et al., 1985; Nhan and Callaway, 2012). Furthermore, the population of neurons that provide feedback to the LGN (Hendrickson et al., 1978), 15\% of the neurons in layer 6 (Fitzpatrick et al., 1994), could be divided into three distinct clusters based on their conduction latency, their maximum response rate, and whether they were simple or complex cells (Briggs and Usrey, 2009). Other earlier findings also indicate the possibility of functional subgroups within the layer 6 population: (1) layer 6 neurons are more likely to be direction-selective than the overall V1 population (Livingstone and Hubel, 1984; Orban et al., 1986; Hawken et al., 1988); (2) they show weak extraclassical receptive field suppression (Schiller et al., 1976a; Gilbert, 1977; Sceniak et al., 2001; Henry et al., 2013); (3) there are distinct populations of layer 6 simple and complex cells (Schiller et al., 1976a; Ringach et al., 2002); and (4) there is a range of contrast sensitivity functions in the layer 6 population indicative of $\mathrm{M}$ - and $\mathrm{P}$-cell pathway dominance (Hawken et al., 1996; Briggs and Usrey, 2009).

In Results, we report that six main functional clusters emerged in the analysis of functional properties of the layer 6 population. The cluster analysis used seven tuning and response measures: (1) orientation bandwidth (obw), (2) direction selectivity (dI), (3) spatial frequency (SF) bandwidth (sfbw), (4) temporal frequency (TF) bandwidth (tfbw), (5) contrast threshold (cTh), (6) the contrast where the response reached $50 \%$ of the maximum (c50), and (7) modulation index ( $\mathrm{fl} / \mathrm{f} 0$ ratio). The results imply that $\mathrm{V} 1$ cortex has very distinct functional classes of neurons within layer 6 , a result that indicates there are multiple parallel circuits within a single cortical hypercolumn, each of which provides information about different features of the visual stimulus. The relative depth of clusters within layer 6 showed that one cluster was located near the top of the layer while others were distributed throughout the layer.

\section{Materials and Methods}

\section{Preparation}

Adult male old-world monkeys (Macaca fascicularis) were used in acute experiments. The animal preparation and recording were performed as described in detail previously (Hawken et al., 1996; Ringach et al., 2002; Xing et al., 2005; Henry and Hawken, 2013). Anesthesia was initially induced using ketamine $(5-20 \mathrm{mg} / \mathrm{kg}$, i.m.) and was maintained with isoflurane (1\%-3\%) during venous cannulation and intubation. For the remainder of the surgery and recording, anesthesia maintained with sufentanil citrate $(6-18 \mu \mathrm{g} / \mathrm{kg} / \mathrm{h}$, i.v.). After surgery was completed, muscle paralysis was induced and maintained with vecuronium bromide (Norcuron, $0.1 \mathrm{mg} / \mathrm{kg} / \mathrm{h}$, i.v.), and anesthetic state was assessed by con- tinuously monitoring the animals' heart rate, EKG, blood pressure, expired $\mathrm{CO}_{2}$, and EEG.

After the completion of each electrode penetration, 3-5 small electrolytic lesions ( $3 \mu \mathrm{A}$ for $3 \mathrm{~s}$ ) were made at separate locations along the electrode track. At the end of the experiments, the animals were deeply anesthetized with sodium pentobarbital $(60 \mathrm{mg} / \mathrm{kg}$, i.v. $)$ and transcardially exsanguinated with heparinized lactated Ringer's solution, followed by $4 \mathrm{~L}$ of chilled fresh $4 \% \mathrm{PFA}$ in $0.1 \mathrm{M} \mathrm{PB}, \mathrm{pH}$ 7.4. The electrolytic lesions were located in the fixed tissue, and electrode tracks were reconstructed to assign the recorded neurons to cortical layers as described previously (Hawken et al., 1988). The borders of layer 6 were defined from combined Nissl and cytochrome oxidase-reacted tissue sections. An example of the Nissl and CO boundaries can be seen in Garcia-Marin et al. (2013, their Fig. $3 E, G)$.

The data from the current study were obtained during the course of the basic characterization of neuronal receptive field properties that is standard for many experiments that we have conducted over the past years where neurons were recorded in all layers of cortex. Some of the data from the current dataset have been published in prior studies, but none has included all of the stimulus dimensions for each neuron that is central to the current study. In 49 animals, $>700$ neurons were recorded in oblique penetrations. A full dataset was obtained for 116 neurons localized to layer 6 .

All experimental procedures were approved by the New York University Animal Welfare Committee and were conducted in compliance with National Institutes of Health's guidelines.

\section{Characterization of visual properties of V1 neurons}

We recorded extracellularly from single units in V1 using glass-coated tungsten microelectrodes. Action potentials were discriminated and recorded as described by Henry and Hawken (2013). Each single neuron was stimulated monocularly through the dominant eye (with the nondominant eye occluded). The steady-state response to drifting gratings was determined to provide characterization of visual response properties. For all neurons included in this study, measurements of orientation tuning, spatial and TF tuning, contrast response with achromatic gratings, and area summation curves were obtained. Receptive fields were located at eccentricities between 1 and 6 degrees. Stimuli were presented using custom software on either a Sony Trinitron GDM-F520 CRT monitor (mean luminance $90-100 \mathrm{~cd} / \mathrm{m}^{2}$ ) or an Iiyama HM204DT-A CRT monitor (mean luminance $60 \mathrm{~cd} / \mathrm{m}^{2}$ ). The monitors' luminance was calibrated using a spectroradiometer (Photo Research PR-650) and linearized via a lookup table using custom software. Stimuli were displayed at a screen resolution of $1024 \times 768$ pixels, a refresh rate of $100 \mathrm{~Hz}$, and a viewing distance of $115 \mathrm{~cm}$.

For all tuning measurements, the responses were recorded to drifting sinusoidal gratings. In the current study, we used the f0 response for neurons with $\mathrm{f} 1 / \mathrm{f} 0$ ratios $<1$ and the $\mathrm{fl}$ response for neurons with $\mathrm{fl} / \mathrm{f} 0$ ratios $>1$. All fitting was done using the MATLAB (The MathWorks) function fmincon where the least-squared error was used to minimize the objective function.

Orientation tuning and direction selectivity. The responses of each neuron were recorded to different orientations between 0 and 360 degrees, either in 20 or 15 degree steps. The stimuli were achromatic gratings at the preferred SF and TF, at a contrast of $\geq 64 \%$. All stimuli were presented in a circular window confined to the classical receptive field (CRF). The measure of orientation selectivity that we used in this study was the bandwidth, which is the width of the smoothed response tuning using the width at $1 / \sqrt{ } 2$ (Schiller et al., 1976a; Ringach et al., 2002). Apart from the use of $\mathrm{f} 0$ responses for neurons with $\mathrm{fl} / \mathrm{f} 0$ ratios $<1$ and the $\mathrm{f} 1$ response for neurons with $\mathrm{f} 1 / \mathrm{f} 0$ ratios $>1$, details are the same as those given in a previous study (Ringach et al., 2002). Direction selectivity (direction index $[\mathrm{dI}]$ ) was determined from the orientation tuning as the response to the preferred orientation and drift direction minus the response to the preferred orientation and opposite drift direction divided by the sum of these two responses.

$S F$ tuning. Each neuron was presented with a range of spatial frequencies, usually in half-octave steps from $<1 \mathrm{c} / \mathrm{deg}$ to $\sim 10 \mathrm{c} / \mathrm{deg}$. For some neurons, the upper limit was extended if the neurons had responses to 
higher spatial frequencies. For almost all neurons that were orientationselective, we measured SF tuning at the preferred orientation and drift direction as well as at the nonpreferred drift direction (see Figs. $1-3 B, G, L, Q$, pairs of filled and unfilled circles). Each set of tuning responses was fit with a difference of offset difference of Gaussians (Hawken and Parker, 1987; Parker and Hawken, 1988) that provides a smooth fit to the data. We obtained the peak SF and the SF bandwidth (width/peak) (Tolhurst and Thompson, 1981) from the fitted functions. For neurons where the response at low SF $(0.1 \mathrm{c} / \mathrm{deg})$ in the fitted function did not go below half the peak we called those cells low pass (lp) in the figures but gave them bandwidth values of 6 in the cluster analysis (see Cluster analysis method).

Contrast response. The response as a function of contrast (RVC) was determined for contrasts ranging from $2 \%$ to $100 \%$ in half-octave steps (see Figs. 1-3C, $H, M, R$ ). A blank condition (screen of mean gray luminance) of the same duration as the stimulus presentation was shown at the beginning of each sequence and interleaved between each contrast presentation. The contrasts were shown in ascending order to avoid adaptation and to minimize hysteresis (Bonds, 1991). Each grating presentation lasted for a minimum of 4 temporal cycles, often more, and was at least $1 \mathrm{~s}$ in duration. Each dataset was fit with a modified Naka-Rushton function (Peirce, 2007) that captured the decrement in response (supersaturation) observed in the contrast response data of some V1 neurons. Two parameters from the fitted contrast response were obtained for each neuron: the contrast threshold, and the contrast (c50) at which the response reached $50 \%$ of the maximum rate. The c50 was determined from the fitted curve; it was not the parameter in the modified Naka-Rushton, the so-called c50 parameter in the equation. The contrast threshold (cTh) was defined as the contrast where the fitted function just exceeded a threshold criterion, the measured spontaneous rate plus 2 SDs of the spontaneous rate. The threshold criterion is drawn as a horizontal dashed line in each example (see Figs. $1-3 C, H, M, R$ ). The point where the criterion intersects the fitted function is the contrast threshold that was used in the current study. The vertical dashed line in each graph marks the threshold. The rightmost short horizontal arrow in each plot is the maximum response of the fitted function. c50 is the point where response reached half the difference between the spontaneous rate and the maximum rate, and it is indicated by the short vertical arrow.

TF tuning. Each neuron was presented with a range of temporal frequencies, usually in 1 octave steps from 0.5 to $32 \mathrm{~Hz}$. Measurements were made for drifting gratings at the optimal orientation and in the preferred and nonpreferred direction (see Figs. $1-3 D, I, N, S$, pairs of filled and unfilled circles). Each set of responses was fit with a difference of exponentials function (Hawken et al., 1996), and from the fitted function to the preferred drift direction we obtained the TF at the maximum response amplitude, the peak TF. In addition, we obtained the TF bandwidth from the preferred direction fitted function, defined as the width of the tuning function when the response was $50 \%$ of the maximum. Neurons where the response at low $\mathrm{TF}(0.1 \mathrm{~Hz})$ in the fitted function did not go below half the peak were called low pass (lp) in the figures but were given bandwidth values of 6 in the cluster analysis (see Cluster analysis methods).

Area summation. The response was measured as a function of stimulus area for a circular patch of grating of optimal SF, TF, orientation, and direction of drift at a contrast of $\sim 90 \%$ of the contrast that produced the maximum response (see Fig. $1 B$ ). Initially, the center of the CRF was estimated by moving an optimal drifting grating with a small aperture (usually 0.1-0.2 degrees in radius) until the experimenter judged that the position of maximum discharge rate had been reached. The patches of grating were centered on the $x-y$ coordinates of the CRF center. A range of sizes from 0.05 or 0.1 degree up to 4 or 5 degrees radius was shown in half octave steps; for a small number of cells tested, the maximum radius was $>5$ degrees $(n=4)$ or 2-3 degrees $(n=9)$ was shown in half-octave steps. Stimuli were shown in pseudorandom order for each repeat of the sequence. The number of repeats was at least three. A blank stimulus of a mean gray screen of the same duration as the stimulus was shown between each repeat. The responses were fit with a difference of Gaussians model (Sceniak et al., 1999, 2001), and the best fitting functions are shown as the smooth curves in Figures $1-3 E, J, O, T$. A measure of area suppression (suppression index [SI]) was obtained from the area tuning as described by Sceniak et al. (2001). The optimal summation radius was determined as the peak of the fitted function. The maximum radius used for the fit was the largest radius tested.

Peak and spontaneous firing rate. The peak firing rate was obtained from each tuning curve. For example, in the orientation domain, the peak rate was the orientation and drift rate that evoked the maximum response. For neurons with $\mathrm{f} 1 / \mathrm{f} 0<1$ (complex cells), the rate was calculated as the average rate across the full duration of the stimulus presentation (f0) while for neurons with $\mathrm{f} 1 / \mathrm{f} 0 \geq 1$ the rate was the amplitude of the first harmonic response (f1). The spontaneous rate for all neurons was the mean rate during the blank (mean gray screen) intervals that were the same duration as the stimulus intervals and that were interleaved with the stimulus presentation.

\section{Cluster analysis method}

The set of tuning and response measures used were those that identify some of the principal emergent properties of cortical neurons, simple/ complex, orientation and direction selectivity, and enhanced selectivity for SF and TF, along with contrast measures that can distinguish $\mathrm{P}$ and $\mathrm{M}$ cell pathways.

Seven response measures and tuning parameters obtained from orientation tuning, SF and TF tuning, and contrast-response measurements were used as the input parameters to the cluster analysis. The parameters used for clustering were f1/f0 ratio, orientation bandwidth, dI, SF bandwidth, TF bandwidth, contrast threshold, and c50 contrast. Each value was converted to a $z$ score for the corresponding distribution. For the three bandwidth measures, if the neuron's tuning was unoriented (in that the response did not drop $<50 \%$ of the maximum response) or low pass in SF or TF, we used values of 120 degrees (orientation bandwidth) and 6 octaves (SF and TF bandwidth) as the values for calculating the $z$ scores. These values are likely to be lower than the real bandwidths of effectively low pass neurons and thus are conservative values. Nonetheless, they allowed us to include the low pass or unoriented tuning functions in the clustering determination. These values are shown as low pass (lp) in the tuning distributions.

The $z$ scores were input to the MATLAB functions pdist and linkage. In linkage, the standard Euclidean distance metric and the unweighted average distance were used in the linkage-clustering algorithm. The output of linkage was used to obtain the hierarchical clusters. With a large set of tuning and response measures, finding the unique optimal number of clusters is not guaranteed. The silhouette function (MATLAB) was applied to the output of the linkage routine to estimate the number of clusters. The silhouette value is determined for each point to measure the distance of that point from its assigned cluster compared with points in other clusters. The value ranges from -1 to 1 where a value close to 1 means the assignment is appropriate. The accumulated sum of silhouette values increases if adding additional clusters provides improved clustering until adding more does not make much difference (i.e., most of the additional points are $\sim 0$ and therefore the accumulated value plateaus), the first plateau can be used as an approximate guide as to the maximum number of clusters to specify in the functions pdist and linkage. In the layer 6 dataset, the plateau was reached with 22 clusters. Six of the clusters contained the majority of the population $(N=85$ of $116,73 \%)$ and appeared to divide the population into distinct clusters.

\section{Experimental design and statistical analysis}

A total of 116 neurons were analyzed for clustering. The results are shown in Tables 1-9. The values for each parameter are given as mean \pm SD in the text and tables. The $n$ for each of the clusters is given in the text. The $p$ values were all determined using a multiple comparison of the output of a one-way ANOVA using the Bonferroni method to provide the adjustment to compensate for multiple comparisons.

\section{Results}

We applied cluster analysis to a population of 116 neurons that we assigned to layer 6 . The methods of layer assignment were described previously (Hawken et al., 1988; Ringach et al., 2002). As described in Materials and Methods, in the cluster analysis, we 
Table 1. Orientation bandwidth

\begin{tabular}{|c|c|c|c|c|c|c|}
\hline & C1 & $\mathrm{C} 2$ & C3 & C4 & $C 5$ & $\overline{C 6}$ \\
\hline $\mathrm{C} 1$ & & 1 & $7.0^{a}$ & $4.9^{a}$ & $5.1^{a}$ & $3.5^{a}$ \\
\hline $29.1 \pm 11.4$ & & & & & & \\
\hline $\begin{array}{l}C 2 \\
26.9 \pm 12.4\end{array}$ & $p=1$ & & $5.6^{a}$ & 3.5 & 3.9 & 1.3 \\
\hline $\begin{array}{l}\text { C } \\
15.9 \pm 5.3\end{array}$ & $p<0.003^{a}$ & $p<0.01^{a}$ & & 1 & 1 & 1 \\
\hline $\begin{array}{l}\text { C4 } \\
17.5 \pm 9.1\end{array}$ & $p<0.03^{a}$ & $p=0.08$ & $p=1$ & & 1 & 1 \\
\hline $\begin{array}{l}C 5 \\
15.4 \pm 4.5\end{array}$ & $p<0.02^{a}$ & $p=0.06$ & $p=1$ & $p=1$ & & 1 \\
\hline $\begin{array}{l}C 6 \\
18.9 \pm 10.5\end{array}$ & $p=0.27$ & $p=0.76$ & $p=1$ & $p=1$ & $p=1$ & \\
\hline
\end{tabular}

The values in the first column under the cluster number show the mean \pm SD for each cluster. The $p$ values (below the diagonal) were all determined using a multiple comparison of the output of a one-way ANOVA using the Bonferroni method to provide the adjustment to compensate for multiple comparisons. Values above the diagonal are $1-\log (p)$ indicating the full range of significance values.

${ }^{a}$ Values reached significance level $<0.05$.

Table 2. Direction index

\begin{tabular}{|c|c|c|c|c|c|c|}
\hline & C1 & $\mathrm{C}$ & C3 & C4 & $C 5$ & C6 \\
\hline C1 & & $39.9^{a}$ & 1 & $26.8^{a}$ & $19.1^{a}$ & $23.1^{a}$ \\
\hline $1.05 \pm 0.30$ & & & & & & \\
\hline $\begin{array}{l}C 2 \\
0.16 \pm 0.14\end{array}$ & $p<0.0001^{a}$ & & $33.7^{a}$ & 2.2 & 2.8 & 1 \\
\hline $\begin{array}{l}\text { C3 } \\
0.90 \pm 0.13\end{array}$ & $p=0.99$ & $p<0.0001^{a}$ & & $20.1^{a}$ & $13.2^{a}$ & $17.4^{a}$ \\
\hline $\begin{array}{l}C 4 \\
0.34 \pm 0.31\end{array}$ & $p<0.0001^{a}$ & $p=0.31$ & $p<0.0001^{a}$ & & 1 & 1 \\
\hline $\begin{array}{l}C 5 \\
0.40 \pm 0.24\end{array}$ & $p<0.0001^{a}$ & $p=0.17$ & $p<0.0001^{a}$ & $p=1$ & & 1 \\
\hline $\begin{array}{l}\text { C6 } \\
0.28 \pm 0.20\end{array}$ & $p<0.0001^{a}$ & $p=1$ & $p<0.0001^{a}$ & $p=1$ & $p=1$ & \\
\hline
\end{tabular}

The values in the first column under the cluster number show the mean \pm SD for each cluster. The $p$ values (below the diagonal) were all determined using a multiple comparison of the output of a one-way ANOVA using the Bonferroni method to provide the adjustment to compensate for multiple comparisons. Values above the diagonal are $1-\log (p)$ indicating the full range of significance values.

${ }^{a}$ Values reached significance level $<0.05$.

used seven tuning parameters: f1/f0 ratio, orientation bandwidth, directional index, SF bandwidth, TF bandwidth, contrast threshold, and c50 contrast. The clustering returned four clusters (C1C4) that each had $\geq 10 \%$ of the neurons and together accounted for $59 \%$ of the population. Two smaller clusters (C5, C6) had $8 \%$ and $7 \%$ of the neurons. It is particularly informative that there were other visual response properties, which were not used as data in the clustering analysis, but were distributed according to the clustering we obtained. Those data will be presented after first examining the tuning curves of clustered neurons on the dimensions used for clustering.

\section{Single-cell examples}

\section{Tuning examples in clusters}

First, we show the distinctive tuning within each of the clusters by presenting examples of the tuning curves of neurons in the six major clusters in the dimensions of stimuli used in the clustering analysis and compare tuning curves between neurons in the major clusters. Also, we present a few examples of tuning curves of neurons from the smaller clusters.

The first main cluster $(\mathrm{C} 1: n=15$ of $116,13 \%)$ was comprised of highly responsive and highly sensitive, direction-selective complex cells (two examples in Fig. $1 A-E, F-J$ ). One distinguishing parameter in the cluster analysis was the $\mathrm{f} 1 / \mathrm{f} 0$ ratio. The $\mathrm{f} 1 / \mathrm{f} 0$ ratios of the two example neurons from the first main cluster were 0.17 and 0.07 , both in the lower quartile of ratios of complex cells. Another major distinguishing feature of the tuning of the first cluster was that all neurons were strongly direction-selective (Fig. 1 $A, F)$. Neurons in the first cluster often showed strong suppression (below the spontaneous baseline shown by the horizontal dotted line) in the nonpreferred direction for a grating of the optimal orientation (e.g., Fig. $1 F$ ). Many of the neurons in C1 also had relatively low contrast thresholds and saturating contrast response functions (Fig. $1 C, H$ ). In the TF domain, most of the neurons in $\mathrm{C} 1$ were strongly bandpass in TF tuning as exemplified in the two example neurons (Fig. 1D,I). In the experiment where the radius of the stimulus window was changed, some neurons in $\mathrm{C} 1$ showed a modest attenuation of their response at the largest window sizes (Fig. $1 E, J$ ), although no parameter from this experiment was used in the cluster analysis.

The neurons in the second major cluster (C2: $n=20$ of 116, $17 \%)$ also had low $\mathrm{f} 1 / \mathrm{f} 0$ ratios $(0.19,0.24$ for the two examples shown in the third and fourth rows of Fig. 1). The neurons in C2 were orientation-selective but not direction-selective (Fig. $1 K, P)$, unlike those in $\mathrm{C} 1$. There were some neurons in $\mathrm{C} 2$ that were low pass in $\mathrm{TF}$ (Fig. $1 \mathrm{~N}$ ) and others that were bandpass (Fig. $1 S$ ). Neurons in C2 showed very little size tuning (Fig. 1O,T).

The neurons in the other four major clusters (C3-C6) had $\mathrm{fl} / \mathrm{f0}$ ratios $>1$, and therefore were classified as simple cells (Skottun et al., 1991). Ratios for the example neurons in C3 (Fig. $2 A-J$ ), C4 (Fig. $2 K-T$ ), C5 (Fig. 3A-E), and C6 (Fig. 3F-J) were $1.5,1.1,1.6,1.5,1.4$, and 1.8 , respectively. The example simple cells in C3 ( $n=18$ of $116,16 \%)$ showed orientation selectivity and strong direction selectivity (Fig. $2 A, F$ ) that were characteristics of this cluster. The strong directional selectivity was observed across all spatial (Fig. $2 B, G$ ) and temporal (Fig. 2D, $I$ ) frequencies tested for the two example neurons. Another distinguishing tuning characteristic of the third cluster is shown by the bandpass TF tuning of the two example neurons (Fig. 2D,I); bandpass TF tuning was also a common feature of this cluster.

The neurons in C4 ( $n=15$ of $116,13 \%)$ were orientationselective but not direction-selective (Fig. $2 K, P$ ). The lack of direction selectivity in $\mathrm{C} 4$ was evident in the spatial (Fig. $2 L, Q$ ) and temporal (Fig. $2 N, S$ ) frequency tuning of the example neurons. The tuning shape for both spatial (Fig. $2 L, Q$ ) and temporal (Fig. $2 N, S$ ) frequency along with the response amplitude was similar in both directions of movement at the preferred orientation. The TF tuning of the two example neurons from cluster 4 were low pass. This was a common but not universal feature of C4. Most of the neurons in clusters 3 and 4 showed little attenuation in their response at large stimulus sizes as shown for the four example neurons (Fig. $2 E, J, O, T$ ).

One distinguishing feature of two additional clusters (C5, $n=$ 9; C6, $n=8$ ) with $\mathrm{fl} / \mathrm{f0}$ ratios $>1$ was their low sensitivity to contrast as can be seen in the nonsaturating contrast response functions from the two example neurons (Fig. $3 C, H$ ). Neurons in C5 and C6 were orientation-selective, and some were moderately direction-selective (Fig. 3A). However, they were distinguished by their TF tuning: neurons in C5 were bandpass in TF (Fig. 3D), whereas those in C6 were low pass in TF (Fig. 3I).

The remaining clusters had few members ( 5 or fewer); therefore, it was not possible to verify their uniqueness with the relatively small population sample. Two features separated most members of these relatively small clusters from the six larger clusters. One was broad orientation tuning. Often neurons had a preferred orientation but also responded across all orientations with a response at the orthogonal-to-preferred orientation (Fig. $3 K, P$ ). The second distinguishing feature was that the broad ori- 
Cluster 1: two example neurons
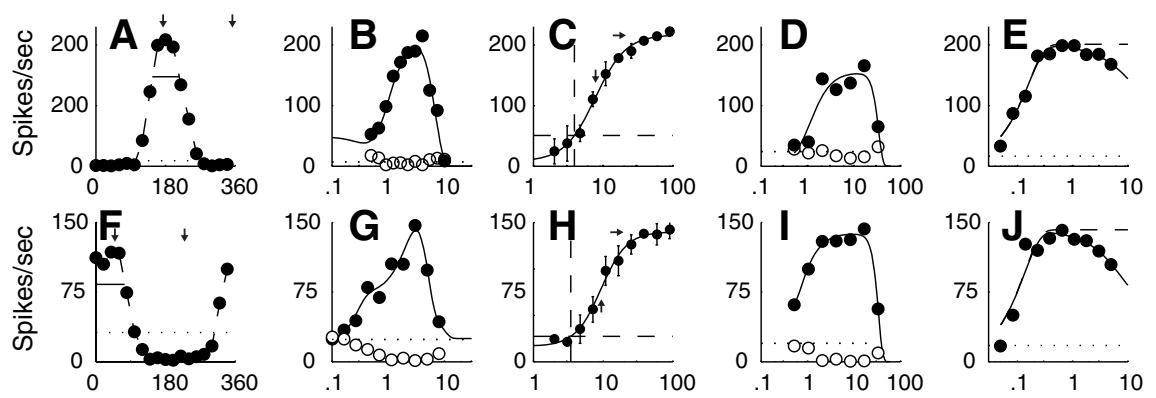

Cluster 2
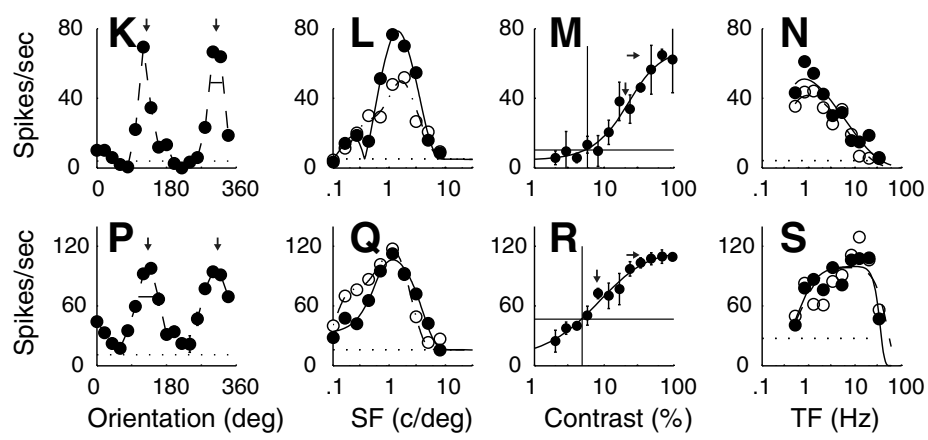

Figure 1. The tuning functions of example neurons from the two main clusters that had $\mathrm{f} 1 / \mathrm{f} 0 \mathrm{ratios}<1$. $\boldsymbol{A}-\boldsymbol{E}$, The tuning from a single neuron in $\mathrm{C} 1(\mathrm{f} 1 / \mathrm{f0}=0.17)$. $\boldsymbol{F}-\boldsymbol{J}$, The tuning for a second neuron in $\mathrm{C} 1(\mathrm{f} 1 / \mathrm{fO}=0.07)$. The neurons in C1 were orientationand direction-selective, had low-contrast thresholds, and were bandpass in TF. There is a high response rate of these neurons, which was a characteristic of this cluster. $\boldsymbol{K}-\mathbf{0}$, The tuning for a neuron in $\mathrm{C}(\mathrm{f} 1 / \mathrm{fO}=0.19)$. $\boldsymbol{P}-\boldsymbol{I}$, The tuning for a second neuron in $\mathrm{C} 2$ ( $\mathrm{f} 1 / \mathrm{f0}=0.24)$. Neurons in this cluster were orientation-selective but not direction-selective, and were mainly low pass in TF $(\boldsymbol{N})$, but some were bandpass $(\boldsymbol{S}) . \boldsymbol{A}, \boldsymbol{F}, \boldsymbol{K}, \boldsymbol{P}$, The response of each neuron was measured as a function of orientation to a drifting achromatic grating at the optimal SF and TF and a high contrast through a window of the optimal size. The pair of small vertical arrows in each plot indicate the optimal orientation that was selected by fitting a von Mises function to the data. Small horizontal bars represent the bandwidth, width at amplitude of $\sqrt{ } 2$ of the maximum. $B, G, L, Q$, The response of each neuron was measured as a function of SF at the preferred orientation and in both drift directions. The pairs of data in each plot indicate the response at the preferred orientation for each drift direction (filled circles represent preferred drift direction; unfilled circles represent nonpreferred drift direction). Smooth curves fitted to the data indicate a spatial receptive field model that is a difference of offset difference of Gaussians (see Materials and Methods). $\boldsymbol{C}, \boldsymbol{H}, \boldsymbol{M}, \boldsymbol{R}$, The response as a function of contrast was measured for each neuron at the preferred orientation, drift direction, SF, and TF in a patch of grating of the optimal diameter. Smooth curve through each dataset indicates a modified Naka-Rushton function (see Materials and Methods). Horizontal dotted line indicates the response that is 2 SDs above the response to a blank gray screen (spontaneous response). Vertical dotted line indicates the value of contrast that was taken as the contrast threshold, the point where the horizontal line intersected the fitted Naka-Rushton function. Error bars indicate SD. $D, I, N, S$, The response as a function of TF is shown for a grating of the optimal orientation, $S F$, and in both the preferred and opposite to preferred direction of drift direction (filled circles represent preferred drift direction; unfilled circles represent nonpreferred drift direction). The two datasets were fitted independently with a difference of exponentials function. Smooth curves indicate the best fitting functions. $\boldsymbol{E}, \boldsymbol{J}, \mathbf{0}, \boldsymbol{T}, \mathrm{A}$ grating of the optimal orientation, drift direction, SF, and TF and at high contrast ( $\geq 64 \%$ ) was presented in a circular window of different radii. The response as a function of radius of the window is shown. Data are fitted with a difference of Gaussians function (see Materials and Methods).

entation tuning was often accompanied by weak bandpass or low pass SF tuning (Fig. 3L, Q).

\section{Population analyses}

We studied how the seven parameters that were used in the clustering were distributed between the different major clusters. Following this, we also studied how surround suppression, peak response rate, and spontaneous rate varied between the clusters.

F1/F0 distribution: simple and complex cells

The $\mathrm{f} 1 / \mathrm{f} 0$ ratio was used as a clustering parameter. $\mathrm{f} 1 / \mathrm{f} 0$ is the ratio of the first-harmonic (f1) modulation amplitude of the response to the elevation of response ( $\mathrm{f} 0$ ). This is a straightforward method of distinguishing simple ( $\mathrm{f} 1 / \mathrm{f0}$ ratio $>1$ ) from complex cells (f1/f0 ratio < 1) (Campbell and Fiorentini, 1973; Movshon et al., 1978; De Valois et al., 1982a,b; Hawken and Parker, 1987;
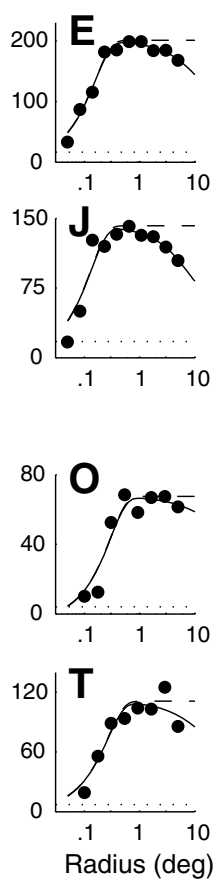

Skottun et al., 1991). All the major clusters that were identified had neurons with either $\mathrm{f} 1 / \mathrm{f} 0<1$ (clusters 1,2 ) or $\mathrm{f} 1 / \mathrm{f} 0>1$ (clusters 3, 4, 5, 6). The distribution of $\mathrm{f} 1 / \mathrm{f} 0$ for the population of layer 6 neurons was bimodal with a very sharp null close to 1 (Fig. 4A).

The $\mathrm{f} 1 / \mathrm{f} 0$ ratio was relatively consistent when determined for the same neuron in different experiments (Fig. 4B). Neurons with $\mathrm{f} 1 / \mathrm{f} 0>1$ (classified as simple) in the orientation tuning experiment (Fig. $4 B, x$ axis) remained so in the subsequent $\mathrm{SF}$ tuning experiment (Fig. $4 B, y$ axis). Only 1 of the 85 of 116 neurons in the major clusters crossed the S/Cx division between experiments; it was classified as a complex cell but segregated with a simple cell cluster. It was a cell with $\mathrm{f} 1 / \mathrm{f} 0=0.8$ that was assigned to cluster 5 where all the other members had $\mathrm{f} 1 / \mathrm{f} 0>1$. In the current dataset, $\mathrm{f} 1 / \mathrm{f} 0$ was obtained from the orientation tuning experiment. When we checked $\mathrm{f} 1 / \mathrm{f} 0$ for this cell in other experiments, f1/f0 was $>1$ ( 1.71 for SF, 1.61 for RVC, 1.65 for TF, and 1.63 for area), so clearly it was on the border. Considering all experiments together, we classified it as a simple cell.

\section{$d I$ and orientation}

bandwidth distributions

Clusters of cells in layer 6 were distinguished by their direction selectivity and also orientation selectivity estimated from orientation tuning bandwidth. Direction selectivity was determined from the $\mathrm{dI}$ (see Materials and Methods). A value of dI near 0 means no preference for direction and dI near 1 means almost completely direction-selective. Neurons that are suppressed in the nonpreferred direction can have a $\mathrm{dI}>1$.

The distribution of $\mathrm{dI}$ in layer 6 was bimodal (Fig. 5B); 39\% (41 of 104) of orientation-selective neurons had a dI $\geq$ 0.8 . There was a population of very direction-selective neurons in layer 6 . Neurons in the orientation- and directionselective complex cell cluster C1 (Fig. 5C, filled black diamonds) had an average dI of $1.05 \pm 0.3$ (mean $\pm \mathrm{SD}$ ), which is significantly different $(p<0.0001)$ from the dI of neurons in the nondirection-selective complex cell cluster $\mathrm{C} 2$ (Fig. $5 C$, filled red circles) in which mean $\mathrm{dI}=0.16 \pm 0.14$. Although $\mathrm{C} 1$ and $\mathrm{C} 2$ differed in $\mathrm{dI}$, they did not have different orientation bandwidth distributions $(29.1 \pm 11.4$ degrees vs $26.9 \pm 12.4$ degrees for $\mathrm{C} 1$ and C2, respectively, $p=1$, not significant). The $\mathrm{dI}$ distributions of the simple cell orientation- and direction-selective cluster, C3 (Fig. 5D, black diamonds), and the non-direction-selective clusters, C4 (Fig. 5D, red circles), C5 (Fig. 5F, green squares), and C6 (Fig. $5 F$, blue diamonds) were significantly different $(p<0.0001$; Table 2 ). All four simple cell clusters had relatively narrow obws (Fig. 5D,F) that were not significantly different between clusters (Table 1). 
Most neurons we studied in layer 6 had a measurable orientation bandwidth, although a small number $(n=12$ of 116 , $10 \%$ ) were nonoriented (Fig. 5A). The distribution of orientation bandwidth for all orientation-selective neurons in layer 6 had a median value of 20.5 deg. (Fig. 5A). Although, when comparing within the same $\mathrm{fl} / \mathrm{f} 0$ clusters, there were no significant differences in orientation bandwidth, there were significant differences between $\mathrm{C} 1$ and $\mathrm{C} 3-\mathrm{C} 5$ and between $\mathrm{C} 2$ and C3 (for $p$ values, see Table 1).

In the population analyses, we show data for the smaller clusters in the figures but do not compare their tuning with the larger clusters in the statistical comparisons (Tables 1-8). The smaller clusters are shown for completeness. We combined 3 small clusters with $\mathrm{fl} / \mathrm{f} 0<1$ and $2<N<$ 9 as solid green symbols for visualization, and show 1 cluster with $\mathrm{f} 1 / \mathrm{f} 0<1, N=4$ as solid blue symbols (Fig. 5D). The 3 clusters with $\mathrm{f} 1 / \mathrm{f} 0>1$ and $2<N<9$ are shown as cyan crosses, purple crosses, and cyan triangles (Fig. 5G). Qualitatively, the cluster with $\mathrm{f} 1 / \mathrm{f} 0<1$ had dIs of $\sim 2$ (Fig. $5 E$, blue filled triangles); these were distinct from all the other neurons as they had relatively high maintained rates and showed a large suppression in the opposite to preferred direction. Other neurons with $\mathrm{f} 1 / \mathrm{f} 0>1$ that were in the minor clusters were relatively untuned for orientation as in the example neurons in Figure $3 K, P$ and accounted for some neurons in the smaller clusters (Fig. 5G).

\section{SF tuning}

One of the characteristic features of many cortical receptive fields is that they have multiple subunits (Hubel and Wiesel, $1962,1968)$ that can result in relatively narrow SF tuning of neurons (Movshon et al., 1978; De Valois et al., 1982a; Hawken and Parker, 1987). We measured the SF tuning at the preferred orientation, drift direction, and TF for high-contrast gratings ( $\geq 64 \%$ contrast) and determined the bandwidth from the difference of offset difference of Gaussians function fitted to the response data (see Materials and Methods). All but two of the neurons in C1 were bandpass in SF (Fig. 6A, black diamonds); the population had mean bandwidth of $2.4 \pm 1.2$ octaves (mean \pm $\mathrm{SD}$ ) and was not significantly different (Table 3) from the bandwidth distribution of neurons in C2 $(2.9 \pm 1.3$ octaves, mean \pm SD) where two neurons were low pass (Fig. 6A, red filled circles). All the neurons in the other four major clusters were bandpass in SF (Fig. 6B, black unfilled di-
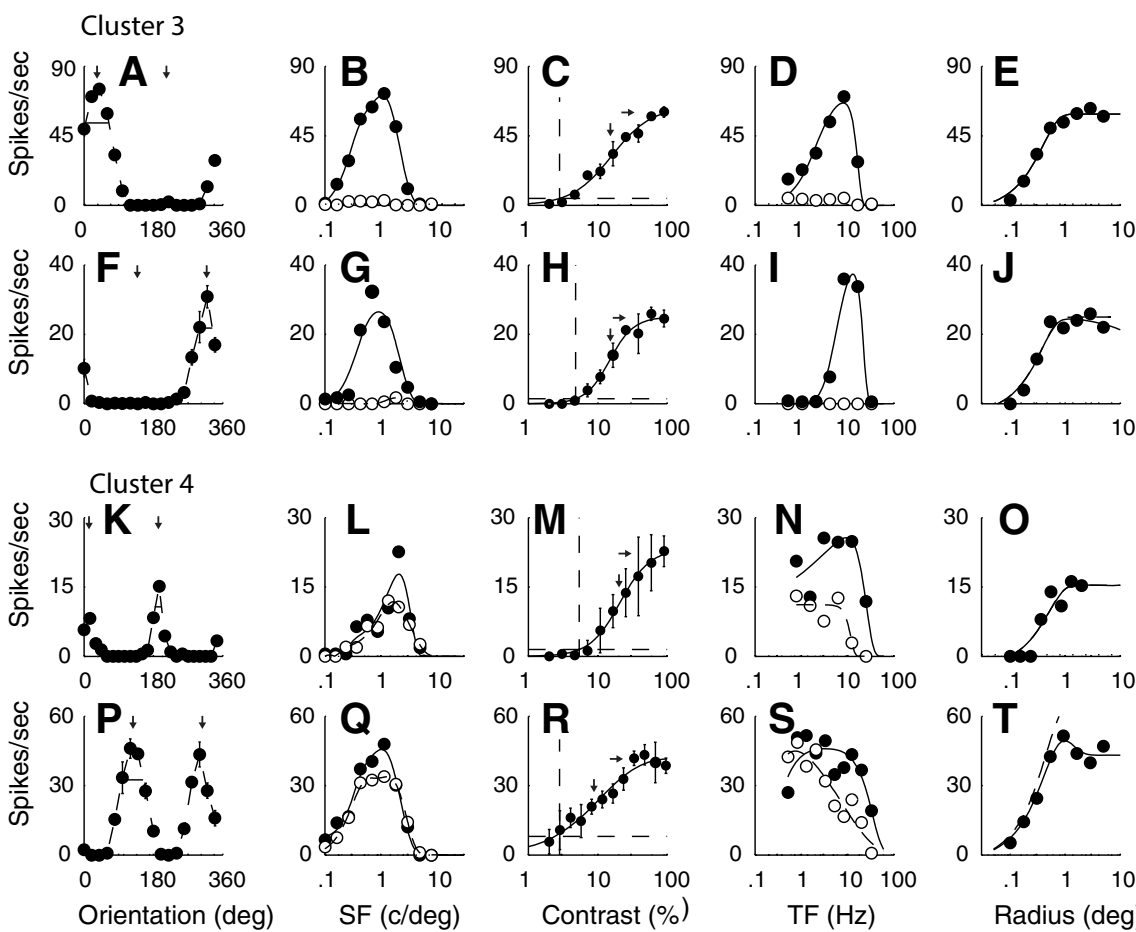

Figure 2. The tuning functions of example neurons from two of the four main clusters that had f1/f0 ratios $>1$. $\boldsymbol{A}-\boldsymbol{E}, \boldsymbol{F}-\boldsymbol{J}$, Tuning of two example neurons from C 3 (f1/f0 1.5 and 1.1, respectively). These neurons, characteristic of the cluster, were strongly direction-selective and bandpass in TF. $\mathbf{K}-\mathbf{O}, \mathbf{P}-\boldsymbol{T}$, Tuning functions of two example neurons from C4 (f1/f0 1.6 and 1.7, respectively). These neurons were orientation-selective but not direction-selective with low pass TF tuning. The other details are as for Figure 1.

\section{Cluster 5}
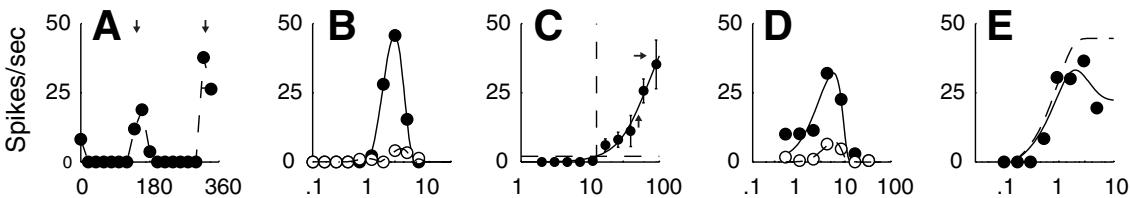

Cluster 6
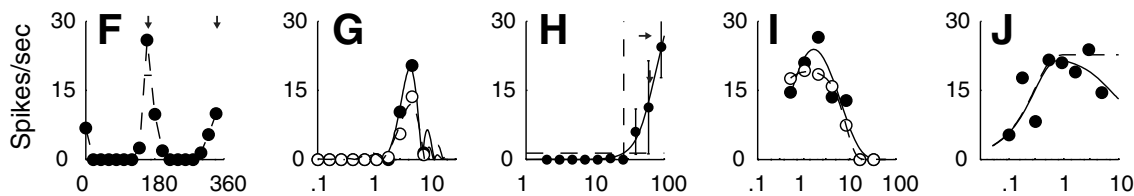

Other Clusters
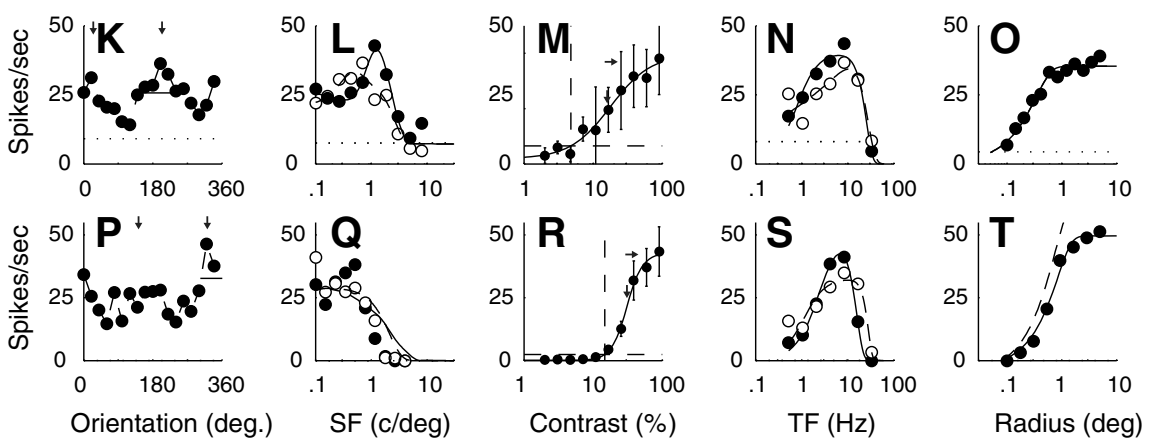

Figure 3. $\boldsymbol{A}-\boldsymbol{E}, \boldsymbol{F}-\boldsymbol{J}$, The tuning functions of example neurons from $\mathbf{C} 5$ and $\mathbf{C 6}$. The neurons in these clusters were orientation and SF selective, but one characteristic that separated them from the other two clusters was their insensitivity to contrast. They are separated from each other by their TF tuning, neurons in $\mathbf{C}$ were bandpass, and those in $\mathbf{C} 6$ were low pass. $\boldsymbol{K}-\mathbf{0}, \boldsymbol{P}-\boldsymbol{T}$, Neurons from the smaller clusters where most of the neurons were weakly selective for orientation and relatively low pass in SF ( $\mathrm{f} 1 / \mathrm{f0} 1.3$ and 1.5, respectively). The other details are as for Figure 1. 

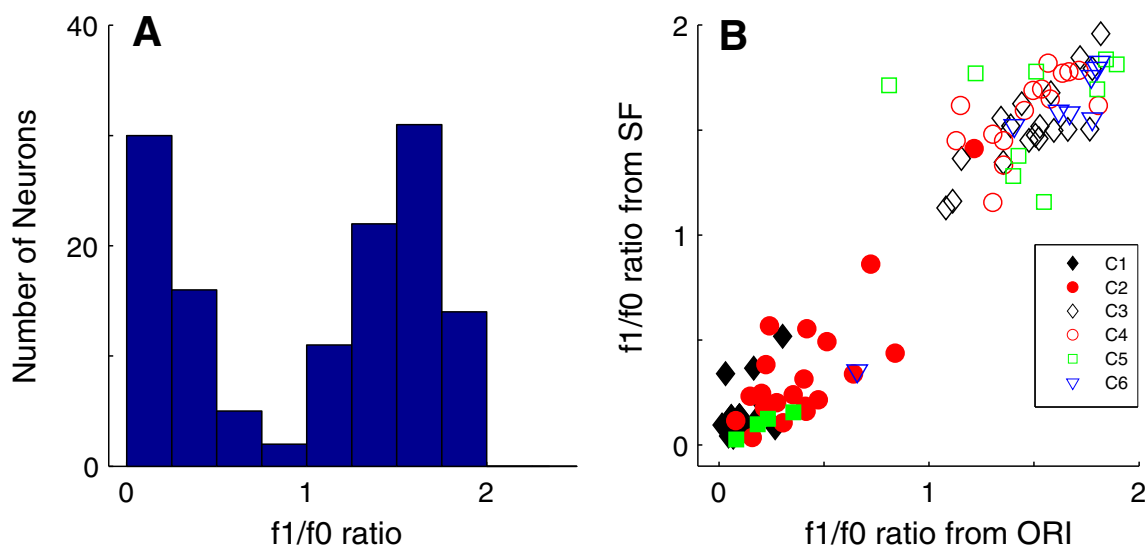

Figure 4. A, The distribution of $\mathrm{f} 1 / \mathrm{fo}$ ratio for all the neurons in the sample from layer $6(n=116)$. The $\mathrm{f} 1 / \mathrm{fo}$ ratio was used as one of the response measures in the clustering procedure. $\boldsymbol{B}$, The $\mathrm{f1} / \mathrm{f0}$ ratio measured in the orientation tuning experiment ( $x$ axis) plotted against the $\mathrm{f1} / \mathrm{f0}$ ratio of the same cell measured from the SF tuning experiment (y axis). Symbols represent cluster assignments. There were six main clusters that are shown as filled black diamonds (C1), filled red circles $(C 2)$, unfilled black diamonds (C3), unfilled red circles (C4), unfilled green squares (C5), and unfilled blue triangles (C6). Unfilled magenta triangles and cyan and magenta crosses represent three smaller clusters with $\mathrm{f1} / \mathrm{f0}$ ratios $>1$. Filled blue triangles represent a small cluster with $\mathrm{f} 1 / \mathrm{f} 0<1$. Filled green squares represent four further clusters with $\mathrm{f} 1 / \mathrm{f0}<1$ and with $<4$ neurons that were combined. The clusters were clearly separated according to whether they are complex (f1/f0 $<1)$ or simple (f1/f0 $>1)$.
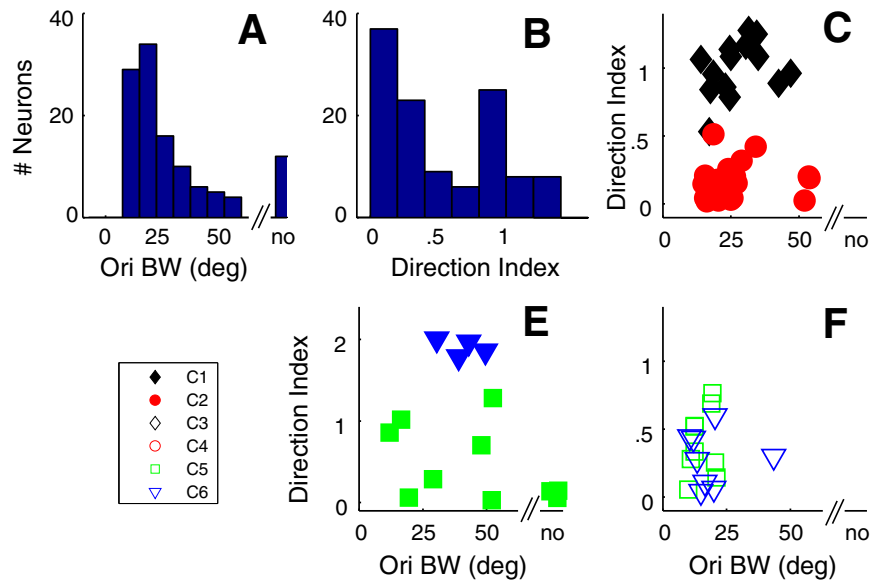

Figure 5. Orientation bandwidth (obw) and dl were two tuning parameters used in the clustering procedure. $\boldsymbol{A}$, The distribution of obw for the whole layer 6 sample. no, Nonoriented. $\boldsymbol{B}$, The distribution of $\mathrm{dl}$ for the whole sample. $\boldsymbol{C}-\boldsymbol{G}$, The relationship between obw ( $x$ axis) and $\mathrm{dl}$ ( $y$ axis) for the same clusters shown in Figure 4. $\boldsymbol{C}$, The obw/dl relationship for C1 (filled black diamonds) and $\mathrm{C}$ (filled red circles). There is a clear separation by dl but not obw in the two clusters. $\boldsymbol{D}$, The relationship for $\mathrm{C}$ (unfilled black diamonds) and (4 (unfilled red circles). There is a clear separation based on dl but not obw in the two clusters. $\boldsymbol{E}$, The obw/dl relationship between the remaining clusters where the clusters also had f1/f0 $<1$. The neurons in the small cluster (filled blue triangles) are very direction-selective and strongly suppressed in the nonpreferred direction. Filled green squares represent the combination of three small clusters. $\boldsymbol{F}$, The obw/dl relationship for $(5$ (green squares) and C6 (blue triangles), both with neurons that have $\mathrm{f1} / \mathrm{f0}>1$. G, Three small clusters (unfilled cyan triangles, magenta crosses, and cyan crosses) where all the clusters also had $\mathrm{f} 1 / \mathrm{fo}<1$.

amonds, C3; red unfilled circles, C4; Fig. 6D, green unfilled squares, C5 and blue triangles, C6). None of their SF bandwidth distributions were significantly different (Table 3 ). The SF bandwidth distribution of $\mathrm{C} 6$ was significantly narrower than the distribution from both $\mathrm{C} 1$ and $\mathrm{C} 2$, whereas the distributions from $\mathrm{C} 3, \mathrm{C} 4$, and $\mathrm{C} 5$ were narrower than C2 (Table 3). Some of the neurons in the smaller clusters were low pass (Fig. 6C,E), whereas others were bandpass (Fig. $6 C$, blue diamonds, E, magenta crosses).

\section{TF tuning}

Previous studies have shown that there is a range of TF tuning in V1. However, there has not been a systematic analysis at the level
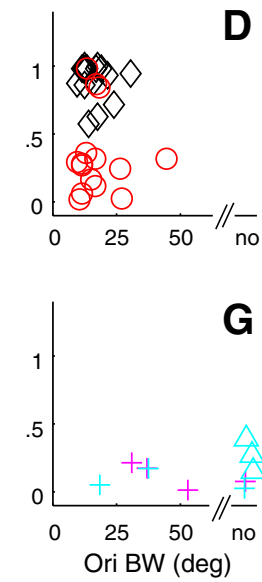

of single cortical layers. In the examples from the different layer 6 clusters, some neurons were strongly bandpass in TF (Figs. 1D,I, 2D,I, 3D), whereas other neurons were low pass in TF (Figs. $1 N$, $2 N, S, 3 I)$. We used TF bandwidth to quantify the width of the tuning and the degree of attenuation at low temporal frequencies. The high- and low-frequency half-height points were determined for each neuron from the fit of a difference of exponentials to the data (see Materials and Methods). If a fit did not attenuate to half-amplitude by a TF of 0.1 , we assigned the low TF half-height point $=0.1$, then used this point and the high frequency at half-amplitude to determine bandwidth. A number of neurons with no or weak and shallow low-frequency attenuation would have a slightly narrower bandwidth than their true bandwidth using this assignment, but we used it to allow us to make quantitative comparisons of the different clusters. Neurons with bandwidths $>$ 6 are shown as low pass (lp) in Figure 7.

Neurons in the main directionselective complex cell cluster (C1) were predominantly bandpass in TF (Fig. 7A, black filled diamonds), whereas the majority of the non-direction-selective complex cell cluster $(\mathrm{C} 2)$ were mostly low pass in their TF tuning (Fig. 7A, red filled circles). The mean bandwidths of the two main complex cell clusters $\mathrm{C} 1$ and $\mathrm{C} 2$ were significantly different $(3.6 \pm 1.9$ vs $5.4 \pm 1.6, p<0.005$; Table 4$)$. There also was a clear separation in the TF bandwidths of the neurons in the two largest simple cell clusters C3 and C4 (Fig. 7B, black unfilled diamonds and red unfilled circles). Neurons in the directionselective cluster (C3) were all bandpass in TF, whereas neurons in the nondirection-selective cluster (C4) were nearly all low pass; the difference between these two clusters was significant $(p<$ 0.0001 ; Table 4 ), as was the difference between C4 and C5 $(p<0.0001)$. However, TF bandwidth distributions of $\mathrm{C} 4$ and $\mathrm{C} 6$ were predominantly low pass (Fig. $7 B$, red circles, $D$, blue triangles) and not significantly different (Table 4). C5 and C6 had significantly different TF bandwidth distributions (Fig. 7D; Table 4).

\section{Contrast response distributions: threshold and c50}

Each neuron's response as a function of contrast for gratings of optimal orientation, direction, and spatial and TF was fit with a modified Naka-Rushton function (Peirce, 2007) (see Materials and Methods). Two measures of the response were obtained from the fitted functions and used as parameters in the clustering: (1) the contrast at which the response reached half its maximum $\left(c_{50}\right)$; and (2) the contrast threshold $\left(\mathrm{c}_{\mathrm{Th}}\right)$, defined as the contrast 
Table 3. Spatial frequency bandwidth

\begin{tabular}{|c|c|c|c|c|c|c|}
\hline & C1 & $\mathrm{C} 2$ & C3 & C4 & $C 5$ & C6 \\
\hline $\mathrm{C} 1$ & & 1 & 1 & 1 & 3.0 & $4.2^{a}$ \\
\hline $2.36 \pm 1.19$ & & & & & & \\
\hline $\begin{array}{l}C 2 \\
2.93 \pm 1.29\end{array}$ & $p=1$ & & $6.3^{a}$ & $5.7^{a}$ & $8.5^{a}$ & $9.7^{a}$ \\
\hline $\begin{array}{l}\text { C } \\
1.80 \pm 0.64\end{array}$ & $p=1$ & $p<0.005^{a}$ & & 1 & 1 & 1 \\
\hline $\begin{array}{l}C 4 \\
1.79 \pm 0.78\end{array}$ & $p=1$ & $p<0.01^{a}$ & $p=1$ & & 1 & 1 \\
\hline $\begin{array}{l}C 5 \\
1.30 \pm 0.34\end{array}$ & $p=0.13$ & $p<0.001^{a}$ & $p=1$ & $p=1$ & & 1 \\
\hline $\begin{array}{l}\mathrm{C} 6 \\
1.10 \pm 0.32\end{array}$ & $p<0.05^{a}$ & $p<0.0005^{a}$ & $p=1$ & $p=1$ & $p=1$ & \\
\hline
\end{tabular}

The values in the first column under the cluster number show the mean \pm SD for each cluster. The $p$ values (below the diagonal) were all determined using a multiple comparison of the output of a one-way ANOVA using the Bonferroni method to provide the adjustment to compensate for multiple comparisons. Values above the diagonal are $1-\log$ indicating the full range of significance values.

${ }^{a}$ Values reached significance level $<0.05$.
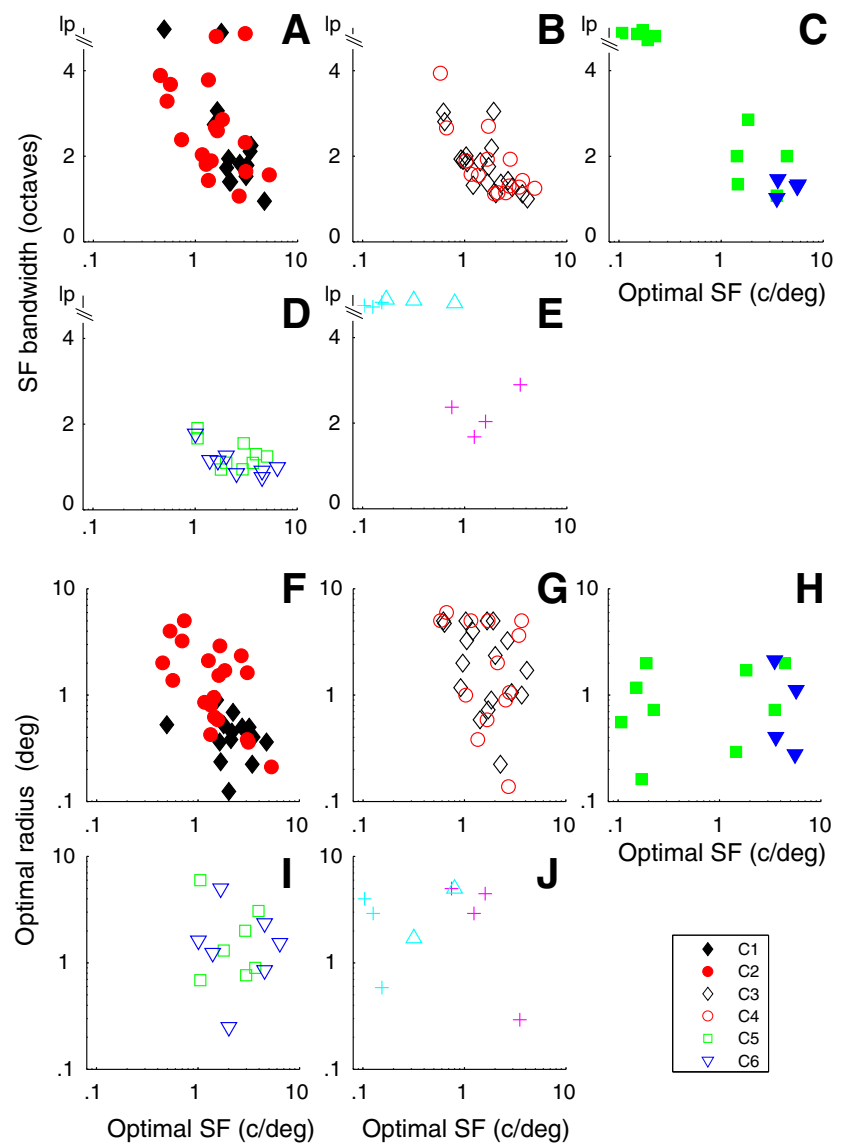

Figure 6. The relationship between optimal SF (osf, $x$ axis) and SF bandwidth (sfbw, $y$ axis, $\boldsymbol{A}-\boldsymbol{E})$ and optimal radius $(\boldsymbol{F}-\boldsymbol{J})$ for the main clusters. Cluster designation as in Figure 5. Ip, Low pass. $\boldsymbol{A}$, In $\mathrm{C} 1$ and $\mathrm{C}$, there is an inverse relationship between osf and sfbw for both groups. $\boldsymbol{B}$, C 3 and $(4$ show overlapping relationships between osf and sfbw. C, The small very directionselective clusters (filled blue triangles) have very similar osfs and sfbws, whereas the combined clusters have a number of neurons that are low pass in SF tuning. D, C5 and C6 show narrow sfbws across a range of osfs. $\boldsymbol{E}$, Neurons in two clusters are low pass in SF; the other is bandpass (magenta crosses). $\boldsymbol{F}-\boldsymbol{J}$, The optimal summation radius for $\mathrm{C} 1$ is restricted, whereas there is a broad span of optimal summation radius for other clusters. The smaller clusters are detailed in Figure $5 C, E, H$, J. For 5 of 85 cells in the 6 main clusters, we did not obtain area summation measures. $\boldsymbol{F}-\boldsymbol{J}$, The $n$ in each cluster $(1-C 6$ was $15,20,18,13,7$, and 7, respectively.
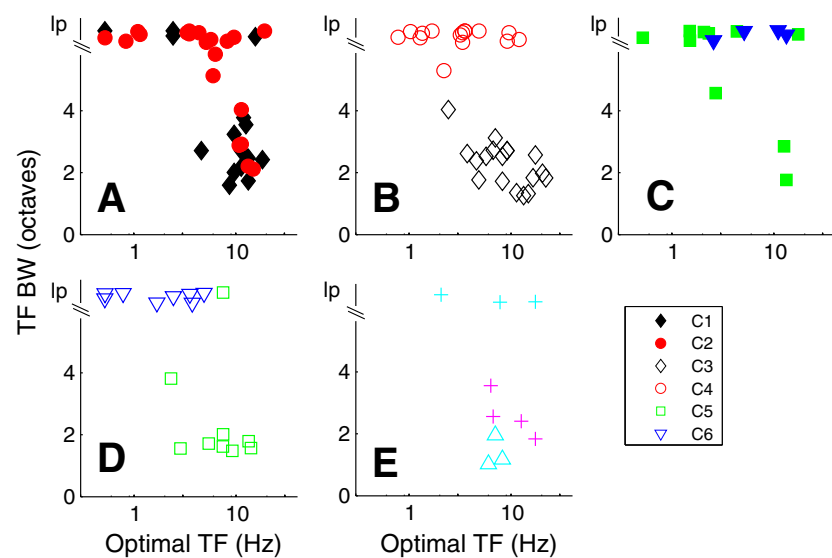

Figure 7. The relationship between optimal TF (otf, $x$ axis) and TF bandwidth (tfbw, $y$ axis) for the main clusters. Cluster designation as in Figure 5. Ip, Low pass. $A, C 1$ and $C 2$, the majority of neurons in $\mathrm{C} 1$ are bandpass in TF and have otfs $\sim 10 \mathrm{~Hz}$. Many of the neurons in cluster 2 are Ip in TF. B, C 3 and C 4 show distinct separation based on tfbw. $C$, The majority of the neurons in the remaining clusters with $\mathrm{f} 1 / \mathrm{f0}$ ratio $<1$ are low pass in TF. $D, C 5$ and $(6$ are separated by their tuning for TF (except 1 neuron in $(5$ that is Ip). $\boldsymbol{E}$, Three small clusters are distinguished by their TF tuning. The smaller clusters are detailed in Figure $5 C, E$.
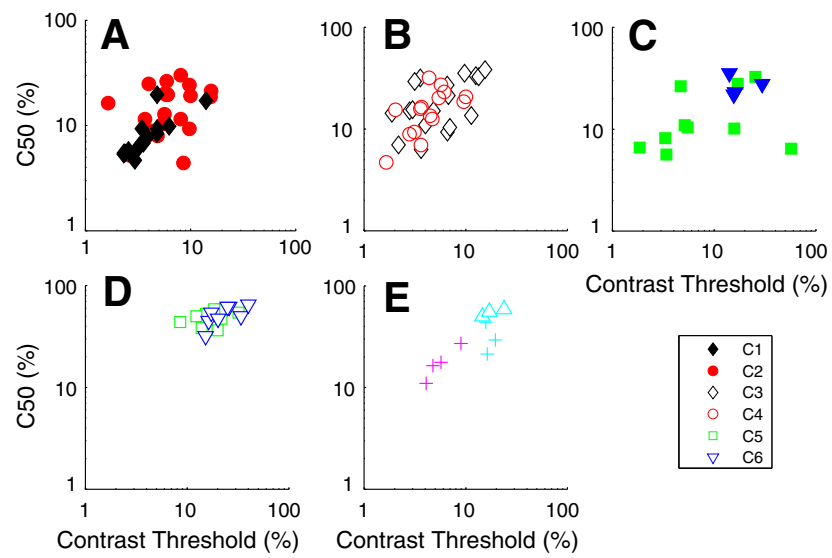

Figure 8. Two measures from the response as a function of contrast were used as tuning parameters in the clustering procedure: contrast threshold (cTh) and the contrast where the response reached half the maximum response (c50). Cluster designation as in Figure 5. $A, C 1$ has neurons with relatively low $C$ Th and $c 50$, whereas neurons in $C 2$ are intermediate in their contrast sensitivity. $\boldsymbol{B}, \mathrm{C} 3$ and C4 have overlapping cThs and c50s but are somewhat less sensitive than C1. $C$, Neurons in the direction-selective cluster (blue triangles) are relatively insensitive to contrast. $\boldsymbol{D}$, Neurons in $C 5$ and $C 6$ have high $c$ Ths and $c 50$, making the clusters stand out as being contrast insensitive. $\boldsymbol{E}$, 0 ne cluster is intermediate in contrast sensitivity (magenta crosses), another insensitive (cyan triangles). The smaller clusters are detailed in Figure 5C, $E$.

where the response exceeded twice the SD of the baseline response. The contrast threshold parameter distributions from clusters C1-C4 (Fig. 8A,B) were not significantly different from each other (Table 5) but were all significantly lower than the parameter distributions from C5 and C6 (Fig. 8D; Table 5).

A similar pattern was found for the $\mathrm{c}_{50}$. The neurons in $\mathrm{C} 5$ and C6 (Fig. $8 D$, green squares and blue diamonds) were relatively insensitive to contrast as seen in the example neurons (Fig. $3 \mathrm{C}, \mathrm{H})$. The mean values of the of $\mathrm{c}_{50}$ 's parameter distributions for C5 and C6 (48.9 $\pm 8.2 \%$ and $52.2 \pm 11.1 \%$, respectively) were not significantly different from each other (Table 6), but they were significantly higher than those of all the other main clusters $(p<0.0001$; Table 6$)$. There was relatively little overlap in the thresholds and $c_{50}$ of C5 and C6 with the other four principal 
Table 4. Temporal frequency bandwidth

\begin{tabular}{|c|c|c|c|c|c|c|}
\hline & C1 & $\mathrm{C} 2$ & C3 & C4 & $C 5$ & C6 \\
\hline $\mathrm{C} 1$ & & $7.4^{a}$ & 3.8 & $14.2^{a}$ & 1.6 & $10.8^{a}$ \\
\hline $3.62 \pm 1.89$ & & & & & & \\
\hline $\begin{array}{l}C 2 \\
5.42 \pm 1.61\end{array}$ & $p<0.005^{a}$ & & $21.5^{a}$ & 1.6 & $13.7^{a}$ & 1.1 \\
\hline $\begin{array}{l}C 3 \\
2.28 \pm 0.71\end{array}$ & $p=0.06$ & $p<0.0001^{a}$ & & $28.4^{a}$ & 1 & $21.8^{a}$ \\
\hline $\begin{array}{l}C 4 \\
6.38 \pm 0.33\end{array}$ & $p<0.0001^{a}$ & $p=0.52$ & $p<0.0001^{a}$ & & $20.0^{a}$ & 1 \\
\hline $\begin{array}{l}C 5 \\
2.46 \pm 1.71\end{array}$ & $p=0.54$ & $p<0.0001^{a}$ & $p=1$ & $p<0.0001^{a}$ & & $16.4^{a}$ \\
\hline $\begin{array}{l}C 6 \\
6.45 \pm 0.13\end{array}$ & $p<0.001^{a}$ & $p=0.95$ & $p<0.0001^{a}$ & $p=1$ & $<0.0001^{a}$ & \\
\hline
\end{tabular}

The values in the first column under the cluster number show the mean \pm SD for each cluster. The $p$ values (below the diagonal) were all determined using a multiple comparison of the output of a one-way ANOVA using the Bonferroni method to provide the adjustment to compensate for multiple comparisons. Values above the diagonal are $1-\log (\mathrm{p})$ indicating the full range of significance values.

${ }^{a}$ Values reached significance level $<0.05$.

Table 5. Contrast threshold

\begin{tabular}{|c|c|c|c|c|c|c|}
\hline & C1 & $C 2$ & C3 & C4 & $C 5$ & C6 \\
\hline C1 & & 1 & 1 & 1 & $19.9^{a}$ & $31.9^{a}$ \\
\hline $4.3 \pm 2.9$ & & & & & & \\
\hline $\begin{array}{l}C 2 \\
7.1 \pm 3.7\end{array}$ & $p=1$ & & 1 & 1 & $15.0^{a}$ & $27.5^{a}$ \\
\hline $\begin{array}{l}\mathrm{C} 3 \\
6.6 \pm 4.2\end{array}$ & $p=1$ & $p=1$ & & 1 & $15.7^{a}$ & $28.0^{a}$ \\
\hline $\begin{array}{l}C 4 \\
4.7 \pm 2.4\end{array}$ & $p=1$ & $p=1$ & $p=1$ & & $18.9^{a}$ & $31.0^{a}$ \\
\hline $\begin{array}{l}C 5 \\
18.2 \pm 6.8\end{array}$ & $p<0.0001^{a}$ & $p<0.0001^{a}$ & $p<0.0001^{a}$ & $p<0.0001^{a}$ & & 2.9 \\
\hline $\begin{array}{l}\mathrm{C} 6 \\
24.2 \pm 8.9\end{array}$ & $p<0.0001^{a}$ & $p<0.0001^{a}$ & $p<0.0001^{a}$ & $p<0.0001^{a}$ & $p=0.15$ & \\
\hline
\end{tabular}

The values in the first column under the cluster number show the mean \pm SD for each cluster. The $p$ values (below the diagonal) were all determined using a multiple comparison of the output of a one-way ANOVA using the Bonferroni method to provide the adjustment to compensate for multiple comparisons. Values above the diagonal are $1-\log (p)$ indicating the full range of significance values.

${ }^{a}$ Values reached significance level $<0.05$.

Table 6. c50 contrast

\begin{tabular}{|c|c|c|c|c|c|c|}
\hline & C1 & $\mathrm{C} 2$ & C3 & C4 & $C 5$ & C6 \\
\hline $\mathrm{C} 1$ & & 3.1 & $7.4^{a}$ & 2.8 & $39.4^{a}$ & $41.4^{a}$ \\
\hline $8.5 \pm 4.4$ & & & & & & \\
\hline $\begin{array}{l}C 2 \\
16.2 \pm 7.4\end{array}$ & $p=0.13$ & & 1 & 1 & $31.8^{a}$ & $34.2^{a}$ \\
\hline $\begin{array}{l}\text { C3 } \\
20.4 \pm 10.8\end{array}$ & $p<0.005^{a}$ & $p=1$ & & 1 & $25.5^{a}$ & $28.2^{a}$ \\
\hline $\begin{array}{l}\text { C4 } \\
16.4 \pm 7.5\end{array}$ & $p=0.18$ & $p=1$ & $p=1$ & & $29.5^{a}$ & $31.9^{a}$ \\
\hline $\begin{array}{l}C 5 \\
48.9 \pm 8.2\end{array}$ & $p<0.0001^{a}$ & $p<0.0001^{a}$ & $p<0.0001^{a}$ & $p<0.0001^{a}$ & & 1 \\
\hline $\begin{array}{l}\text { C6 } \\
52.2 \pm 11.1\end{array}$ & $p<0.0001^{a}$ & $p<0.0001^{a}$ & $p<0.0001^{a}$ & $p<0.0001^{a}$ & $p=1$ & \\
\hline
\end{tabular}

The values in the first column under the cluster number show the mean \pm SD for each cluster. The $p$ values (below the diagonal) were all determined using a multiple comparison of the output of a one-way ANOVA using the Bonferroni method to provide the adjustment to compensate for multiple comparisons. Values above the diagonal are $1-\log (p)$ indicating the full range of significance values.

${ }^{a}$ Values reached significance level $<0.05$.

clusters. Among the other four clusters, only the $c_{50}$ parameter distribution for $\mathrm{C} 3$ was significantly higher than for $\mathrm{C} 1$ (Table 6). The smaller simple cell clusters had restricted ranges of $c_{50}$ and $\mathrm{C}_{\mathrm{Th}}$ values (Fig. $8 E$ ) within the clusters but were within the range of values in the major clusters.
Table 7. Peak firing rate

\begin{tabular}{lllllll}
\hline & $C 1$ & $C 2$ & $C 3$ & $C 4$ & $C 5$ & $C 6$ \\
\hline$C 1$ & & $12.3^{a}$ & $13.2^{a}$ & $12.3^{a}$ & $10.5^{a}$ & $9.9^{a}$ \\
$140 \pm 43$ & & & & & & \\
$C 2$ & $p<0.0001^{a}$ & & 3.7 & 5.7 & 7.8 & 8.5 \\
$52 \pm 19$ & & & & 2.5 & 4.8 & 5.6 \\
$C 3$ & $p<0.0001^{a}$ & $p=1$ & & & & \\
$37 \pm 16$ & & & & & 3.1 & 3.0 \\
$C 4$ & $p<0.0001^{a}$ & $p=0.38$ & $p=1$ & & & \\
$29 \pm 14$ & & & & & & \\
$C 5$ & $p<0.0001^{a}$ & $p<0.07$ & $p=1$ & $p=1$ & & \\
$19 \pm 9$ & & & & & & \\
$C 6$ & $p<0.0001^{a}$ & $p=0.06$ & $p=1$ & $p=0.14$ & $p=0.85$ & \\
$18 \pm 7$ & & & & & &
\end{tabular}

The values in the first column under the cluster number show the mean \pm SD for each cluster. The $p$ values were all determined using Kruskal-Wallis nonparametric test.

${ }^{a}$ Values reached significance level $<0.05$.

Table 8. Spontaneous firing rate

\begin{tabular}{|c|c|c|c|c|c|c|}
\hline & C1 & $\mathrm{C} 2$ & C3 & C4 & $C 5$ & C6 \\
\hline $\begin{array}{l}\mathrm{C} 1 \\
14.0 \pm 7.3\end{array}$ & & 3.6 & $15.1^{a}$ & $13.3^{a}$ & $10.5^{a}$ & $10.1^{a}$ \\
\hline $\begin{array}{l}C 2 \\
9.0 \pm 6.3\end{array}$ & $p=0.07$ & & $16.2^{a}$ & $13.4^{a}$ & $10.7^{a}$ & $10.7^{a}$ \\
\hline $\begin{array}{l}\text { C3 } \\
0.10 \pm 0.20\end{array}$ & $p<0.0001^{a}$ & $p<0.0001^{a}$ & & 2.6 & 2.4 & 2.1 \\
\hline $\begin{array}{l}C 4 \\
0.51 \pm 1.53\end{array}$ & $p<0.0001^{a}$ & $p<0.0001^{a}$ & $p=0.20$ & & 1.1 & 1.2 \\
\hline $\begin{array}{l}C 5 \\
0.36 \pm 0.82\end{array}$ & $p<0.0001^{a}$ & $p<0.0001^{a}$ & $p=0.24$ & $p=0.88$ & & 1.2 \\
\hline $\begin{array}{l}\mathrm{C} 6 \\
0.09 \pm 0.14\end{array}$ & $p<0.0001^{a}$ & $p<0.0001^{a}$ & $p=0.32$ & $p=0.79$ & $p=0.85$ & \\
\hline
\end{tabular}

The values in the first column under the cluster number show the mean \pm SD for each cluster. The $p$ values were al determined using Kruskal-Wallis nonparametric test.

${ }^{a}$ Values reached significance level $<0.05$.

Table 9. Area suppression index

\begin{tabular}{|c|c|c|c|c|c|c|}
\hline & C1 & $C 2$ & C3 & C4 & $C 5$ & C6 \\
\hline $\mathrm{C} 1$ & & $5.0^{a}$ & $7.1^{a}$ & $6.4^{a}$ & $5.1^{a}$ & $4.3^{a}$ \\
\hline $0.38 \pm 0.22$ & & & & & & \\
\hline $\begin{array}{l}C 2 \\
0.14 \pm 0.18\end{array}$ & $p<0.025^{a}$ & & 1.3 & 1.2 & 1.0 & 1.1 \\
\hline $\begin{array}{l}\mathrm{C} 3 \\
0.11 \pm 0.24\end{array}$ & $p<0.025^{a}$ & $p=0.73$ & & 1.0 & 1.2 & 1.3 \\
\hline $\begin{array}{l}\text { (4) } \\
0.12 \pm 0.22\end{array}$ & $p<0.005^{a}$ & $p=0.78$ & $p=0.96$ & & 1.2 & 1.2 \\
\hline $\begin{array}{l}C 5 \\
0.14 \pm 0.16\end{array}$ & $p<0.025^{a}$ & $p=0.99$ & $p=0.80$ & $p=0.83$ & & 1.1 \\
\hline $\begin{array}{l}\text { C6 } \\
0.14 \pm 0.25\end{array}$ & $p<0.05^{a}$ & $p=0.92$ & $p=0.77$ & $p=0.79$ & $p=0.95$ & \\
\hline
\end{tabular}

The values in the first column under the cluster number show the mean + SD for each cluster. The $p$ values were determined using Student's $t$ test. For 5 of 85 cells in the 6 main clusters, we did not obtain area summation measures. The $n$ in each cluster $(1-C 6$ was $15,20,18,13,7$, and 7, respectively.

${ }^{a}$ Values reached significance level $<0.05$.

Area tuning distributions

Most neurons in our sample summed visually evoked signals as stimulus size increased until a maximal area (Figs. 1-3 E,J,O,T) and then maintained a plateau, with a small amount of suppression at the largest areas for a few neurons. There was a relatively broad distribution of optimal radius within the most of the major clusters (Fig. $6 F-J$ ), although $\mathrm{C} 1$ showed a narrower range, probably due to the small degree of surround suppression. Areal summation with weak or no surround suppression is characteristic of layer 6 neurons' receptive fields (Schiller et al., 1976a; Gilbert, 


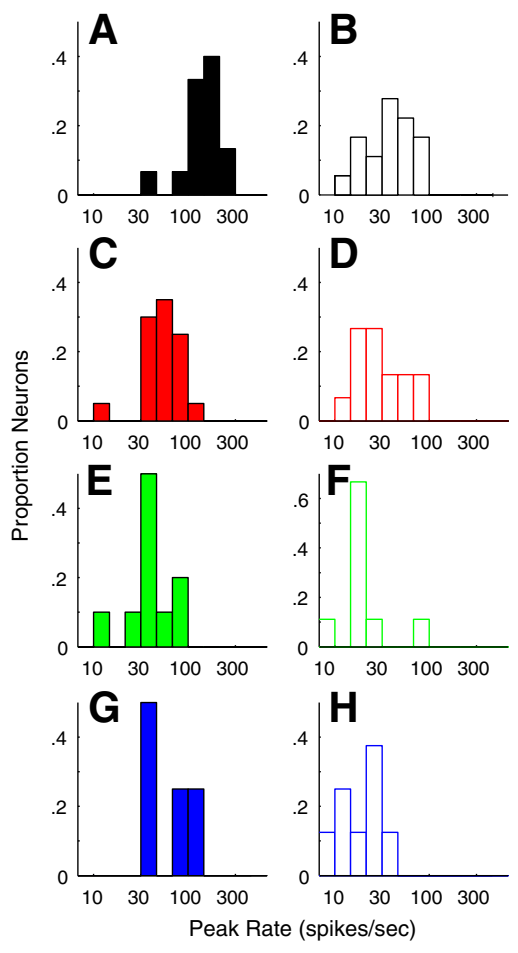

Figure 9. Distribution of maximum firing rates (see Materials and Methods) to optimal stimuli in each cluster. $A, C, E, G$, The distributions of peak firing rate of neurons in $\mathrm{C1}, \mathrm{C} 2, \mathrm{C} 6$, and $C 7$, respectively, all that had $\mathrm{f} 1 / \mathrm{fo}$ ratios $<1$. Many neurons in $\mathrm{C} 1$ have high maximum firing rates. $\boldsymbol{B}, \boldsymbol{D}, \boldsymbol{F}, \boldsymbol{H}$, The distributions of peak firing rate of neurons in $\mathrm{C} 3, \mathrm{C} 4, \mathrm{C} 5$, and $(6$ respectively, all had $\mathrm{f} 1 / \mathrm{f0}$ ratios $>1$.

1977). The only cluster that showed a consistent pattern of suppression, albeit relatively weak (mean SI $=0.38 \pm 0.22$; Table 9), at the largest diameters was $\mathrm{C} 1$. The suppression observed in $\mathrm{C} 1$ neurons was significantly greater than in all the other clusters (Table 9). In the area measurements, the maximum stimulus radius tested was 4 or 5 degrees for the majority of neurons. Therefore, if there were additional attenuation beyond this spatial extent caused by surround suppression, it would not have been measured. As a consequence, suppression may have been underestimated if it extended beyond a radius of 5 degrees.

Response rate and spontaneous rate

Seven functional tuning properties were extracted and $z$-transformed for the clustering, but there were other tuning measures and functional response properties that were not used. A number of these are particularly notable because they tended to distribute according to cluster, even though they were not included in the clustering analysis.

Consider maximum spike rate. Across the six main clusters, there were mean maximum rates ranging from 140 spikes/s down to 18 spikes/s. The two main clusters accounting for the complex cell population $(\mathrm{f} 1 / \mathrm{f} 0<1$ ) had clearly different ranges of peak firing rates. In the direction-selective complex cell cluster $(\mathrm{C} 1)$, the mean rate was $140 \pm 43$ spikes/s (Fig. 9A), which was significantly different from the mean rate of $52 \pm 19$ spikes/s (Fig. 9C) in cluster C2 ( $p<0.0001$; Table 7$)$.

The remaining four major clusters were predominantly simple cells. The larger two of these clusters (C3 and C4) had mean maximum spike rates $37 \pm 16$ and $29 \pm 14$ spikes/s, respectively, which were not significantly different $(p=0.26)$, whereas the two smaller clusters, C5 and C6, had mean peak rates of $19 \pm 9$ and $18 \pm 7$ spikes/s, respectively (compare Table 7).

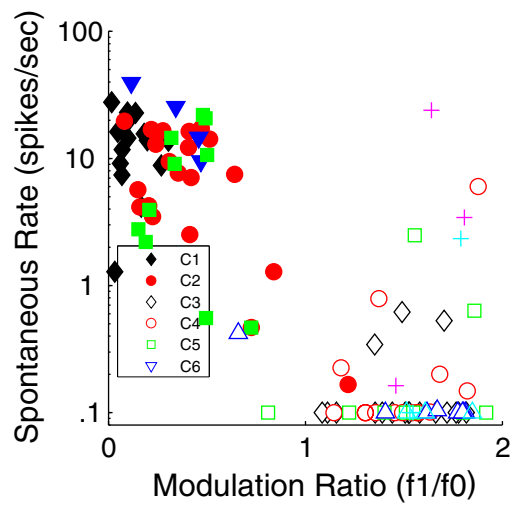

Figure 10. Distribution of spontaneous firing rates (y axis) as a function of $f 1 / f 0$ ratio ( $x$ axis) for all neurons shown as clusters in Figure 4 . Neurons with spontaneous rate of $\leq 0.1$ are shown as 0.1 . There are two main groups: those with $\mathrm{f1} / \mathrm{f0}<1$ have spontaneous rates $>1$, and those with $\mathrm{f} 1 / \mathrm{fo}>1$ have low spontaneous rate. Clusters as in Figure 4. The smaller clusters detailed in the Figure 5 legend are shown with the same symbols used in Figure 5.

It is well established that spontaneous firing rate differs between simple and complex cell populations in the infragranular layers of macaque V1 (Schiller et al., 1976a,b; Ringach et al., 2002). We determined that this was the case for the layer 6 population (Fig. 10). The mean spontaneous rate for all neurons with $\mathrm{f} 1 / \mathrm{f} 0$ ratios $<1$ (complex cells) was $11.2 \pm 8.2$ spikes/s, whereas the mean rate for all neurons with $\mathrm{fl} / \mathrm{f} 0$ ratios $>1$ (simple cells) was $1.2 \pm 3.7$ spikes/s. These distributions were significantly different $(p<0.0001)$. C1 had the highest spontaneous rate (14 \pm $7.3 \mathrm{spikes} / \mathrm{s})$. C1's spontaneous rate was not significantly different from the rate of C2 ( $9 \pm 6.3$ spikes/s; Table 8$)$. Of the overall population of neurons with $\mathrm{f} 1 / \mathrm{f} 0$ ratios $>1$, the four major simple cell clusters (C3-C6) all had similar ranges of $\mathrm{f1} / \mathrm{f} 0$ and also had low mean spontaneous rates, all $<1$ spike/s (Table 8 ) that did not differ between the clusters.

\section{Relative depth of clusters within layer 6}

We determined whether the position of the recorded neurons within each cluster was distributed evenly across the depth of layer 6 or whether there was any localization within the layer. This analysis was done by ordering the neurons in each cluster according to their relative depth within layer 6 , and then comparing the resulting profile with the profile of the total population. The neurons in $\mathrm{C} 1$ were all found in the upper half of the layer as can be seen by comparing the black diamonds $(\mathrm{C} 1)$ with the small blue circles (total population) in Figure $11 \mathrm{~A}$. In contrast, the neurons in C2 (Fig. 11A, red circles) were distributed through the depth of the layer. There did not appear to be any major aggregation of neurons in the other main clusters (C3-C6) in the upper or lower regions of layer 6 (Fig. 11B).

\section{Discussion}

\section{Cluster analysis results}

Of the seven tuning features used for cluster analysis, three measures were particularly useful for distinguishing the six major functional clusters in layer 6: modulation ratio ( $\mathrm{f} 1 / \mathrm{f} 0)$, directional selectivity, and TF tuning.

The neurons in two of the six major clusters ( $\mathrm{C} 1$ and $\mathrm{C} 2$ ) had $\mathrm{f} 1 / \mathrm{f} 0$ ratios $<1$ and would be classified as complex cells (Skottun et al., 1991). Neurons in the other four clusters (C3-C6) had f1/f0 ratios $>1$ and would be classified as simple cells.

A second feature defining the clusters was direction selectivity. There were two major clusters, C1 and C3, that had neurons that 

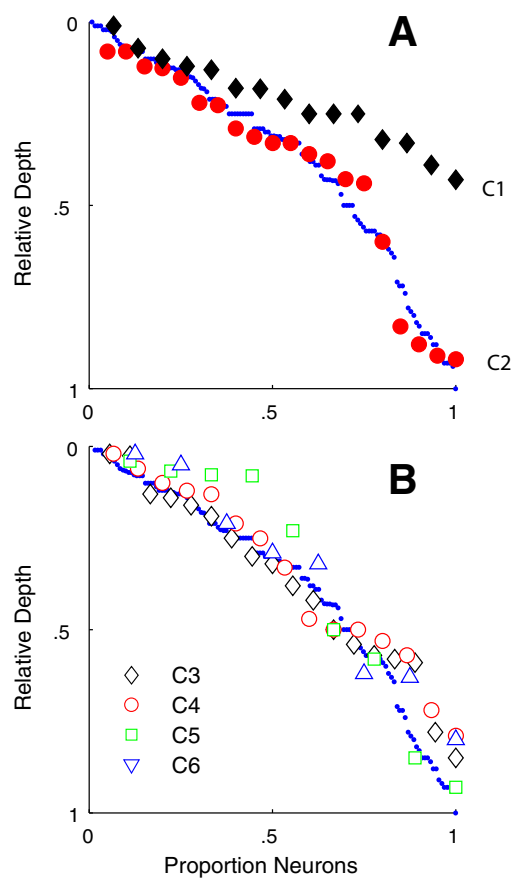

Figure 11. The relative depth of each neuron is shown in serial order from top of layer 6 . Each cluster is shown by the same symbols as used in Figure 4 and subsequent figures. $A$, The relative depth for $\mathrm{C} 1$ (black diamonds) shows that the neurons are found in the upper half of layer 6 . Neurons in $C 2$ (red circles) are distributed through the full depth of layer 6 . Small blue circles represent the serial order of the total sample of 116 neurons. $\boldsymbol{B}$, The four clusters with $\mathrm{f} 1 / \mathrm{f0}$ ratios $>1, C 3-C 6$, are distributed through the full depth of layer 6 .

were strongly direction-selective (Fig. $5 A, B$, filled and unfilled black diamonds).

A third tuning measure, TF bandwidth, was also important in distinguishing $\mathrm{C} 1$ and $\mathrm{C} 3$ from the non-direction-selective complex and simple cell clusters. $\mathrm{C} 1$ and $\mathrm{C} 3$ were bandpass in TF (Figs. $7 A, B, 12$, filled and unfilled black diamonds). Three of the remaining four major clusters $(\mathrm{C} 2, \mathrm{C} 4$, and $\mathrm{C} 6)$ were either complex or simple (Figs. $4 B, 12$ ), but they were non-directionselective (Figs. $5 A, B, 12$ ) and mostly low pass in TF (Figs. $7 A, B$, 12).

\section{Tuning and response measures not used in the}

\section{cluster analysis}

Although we did not use firing rate as a parameter for clustering, we showed (in Results) that visually driven firing rate was much higher in $\mathrm{C} 1$ than in $\mathrm{C} 2$, and both clusters $\mathrm{C} 1$ and $\mathrm{C} 2$ had higher average firing rates than in the simple cell clusters C3-C6. Similarly, spontaneous firing rate was significantly higher in $\mathrm{C} 1$ and $\mathrm{C} 2$ than in $\mathrm{C} 3-\mathrm{C} 6$, and the separation between complex and simple cells within layer 6 (Fig. 10) was even more evident than the separation in the overall V1 population (Ringach et al., 2002). Furthermore, the distribution of cells in depth was different for C1 from that of all the other clusters (Fig. 11).

\section{Properties and possible functions of neurons in different layer 6 clusters \\ Cluster 1}

The visually evoked mean rate was 140 spikes/s for C1 neurons, and they did not appear to adapt, much like the rates of PV inhibitory interneurons (Rudy and McBain, 2001). The nonadapting high spike rate of the PV inhibitory interneurons is attributed to the expression of the Kv3.1b potassium channel

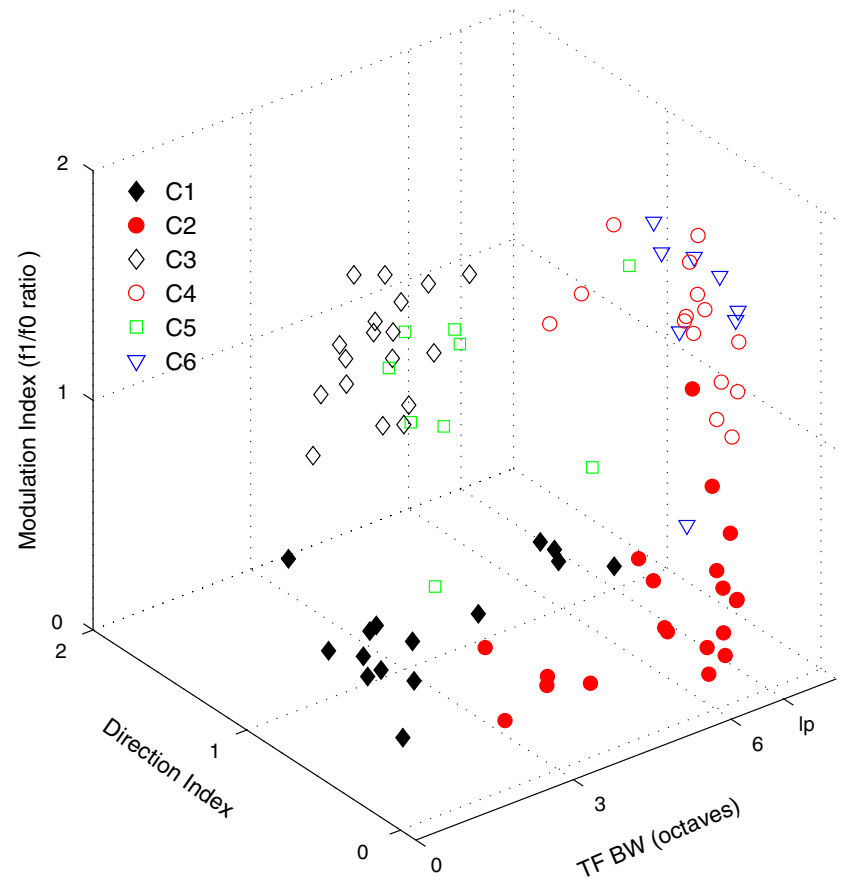

Figure 12. Distribution of the six largest clusters is shown with respect to three tuning parameters: TF bandwidth ( $x$ axis), f1/f0 ratio ( $y$ axis), and dl ( $z$ axis). Each cluster is indicated by the symbols described in Figure 4.

(Rudy and McBain, 2001). In rodents, the Kv3.1b channel is not expressed in excitatory neurons in cortex. However, it is expressed in some excitatory neurons in primate cortex (Constantinople et al., 2009; Kelly et al., 2019), and it is clearly expressed in the large Meynert cells located near the layer 5/6 border in monkey visual cortex (Ichinohe et al., 2004; Constantinople et al., 2009; Kelly et al., 2019). It is tempting to speculate that some of the high firing rate neurons in $\mathrm{C} 1$ are large pyramidal neurons that express Kv3.1b. All neurons in C1 were located in the top half of the layer, layer 6a (Fig. 11). Because Meynert cells are sparse, it is unlikely that all the neurons in $\mathrm{C} 1$ are Meynert cells.

Neurons in C1 were very direction-selective (Figs. 5C, 12). It has been established that most Meynert cells project to extrastriate visual area MT (Fries et al., 1985; Nhan and Callaway, 2012), which contains many direction-selective neurons (Zeki, 1974). Further, some of the tuning characteristics of the neurons in $\mathrm{C} 1$, such as strong directionality, low contrast threshold (Fig. 8A), and saturating contrast response functions (Fig. $1 C, H$ ), match those of the neurons described by Movshon and Newsome (1996) that were recorded in layer 6 of macaque V1 and that were activated antidromically by electrical stimulation of MT. The neurons in $\mathrm{C} 1$ showed some response attenuation at large stimulus window diameters (Fig. 1E,J) indicative of eCRF suppression. Their eCRF suppression may be inherited from magnocellular LGN neurons because M-pathway retinal ganglion cells (presumptive parasol cells) show moderate eCRF suppression (Solomon et al., 2006). If some of the $\mathrm{C} 1$ population are also providing input to MT, it is consistent with the findings that the functional characteristics of the input to MT neurons are derived mainly via V1, V2, and V3 through the M-pathway (Gegenfurtner et al., 1994).

\section{Cluster 2}

Neurons in C2 were also complex cells ( $\mathrm{f} 1 / \mathrm{f} 0$ ratio $<1)$ but were not direction-selective (Fig. $1 K, P$ ). However, all $\mathrm{C} 2$ neurons 
were orientation- and SF-selective (Fig. $1 L, Q$ ). The mean spike rate of the neurons in $\mathrm{C} 2$ was significantly lower than the mean rate in $\mathrm{C} 1$ (Table 7). As a cluster, they had higher average $\mathrm{CTh}$ and c50 values than $\mathrm{C} 1$ (Fig. 8; Tables 5, 6), but the differences were not significant. Sixty-five percent of the neurons in $\mathrm{C} 2$ were low pass in TF (13 of 20; Fig. $1 N$ ), and the majority showed little eCRF suppression: the mean SI was 0.2 , but there were two neurons that had SI $>0.7$. In distinction to the neurons in $\mathrm{C} 1$, this group of neurons would provide signals about stationary or slowly moving borders.

\section{Cluster 3}

All the neurons in $\mathrm{C} 3$ were simple cells $(\mathrm{f} 1 / \mathrm{f} 0$ ratio $>1$, mean $=$ $1.5)$ that were direction-, orientation-, and SF-selective and often strongly bandpass in TF (Fig. $2 A-J$ ). The average orientation bandwidth was 16 degrees (Fig. 5D; Table 1), showing the narrowest average tuning of all the clusters. The neurons in $\mathrm{C} 3$ also had narrow TF bandwidths (mean 2.3 octaves; Table 4) but a range of optimal TFs (Fig. $7 B$ ). The combination of narrow tuning for TF, but a range of optimal TFs and a range of optimal SFs (Fig. 6B), suggests that this population of simple cells may span a selective range of velocity preferences. This cluster of neurons would signal the sign and direction of movement of objects and edges over all but the lowest contrast range.

\section{Cluster 4}

The neurons in $\mathrm{C} 4$ were distinguished from those of $\mathrm{C} 3$ by their lack of direction selectivity (Figs. $2 K, L, N, P, Q, S$, Fig. $5 D$ ) and their low pass TF tuning (Fig. $7 B$; Table 4). The cluster had a similar range of contrast threshold and c50 to C3. The average peak firing rate of neurons in $\mathrm{C} 4$ was not significantly different from the rate in $\mathrm{C} 3$ (Table 7 ). The neurons in $\mathrm{C} 4$ were significantly more sensitive to contrast than those in C5 and C6 (Tables $5,6)$. This cluster of neurons, because they are simple cells and are low pass in TF, are the only cluster that would signal the sign and location of oriented edges in an image across a range of sizes at low and intermediate contrasts in a stationary achromatic image.

\section{Clusters 5 and 6}

Most neurons in C5 and C6 were characterized by relatively narrow orientation and SF bandwidth combined with low sensitivity to achromatic contrast. It has been a longstanding mystery how the visual system maintains a high level of contrast discrimination for achromatic stimuli over a wide range of base contrasts (Legge, 1981; Barlow et al., 1987; Chirimuuta and Tolhurst, 2005a,b). The parcellation of neurons into clusters with different dynamic ranges for different contrast ranges suggests a solution to this long-standing puzzle of contrast discrimination and identification (Chirimuuta and Tolhurst, 2005a,b). Clusters C5 and C6 were distinguished from each other by their TF selectivity. Almost all neurons in C5 were bandpass for TF (Fig. 7D, green squares), whereas those in C6 were low pass for TF (Fig. 7D, blue triangles). Both clusters were distributed through the depth of layer 6 (Fig. 11B).

\section{Minor clusters}

Some of the smaller clusters had neurons that were relatively untuned for orientation and low pass in SF (Figs. $3 K, L, P, Q$, $5 E, G, 6 C, E)$. These are properties that have been attributed to inhibitory interneurons (Nowak et al., 2008), but anatomical identification along with functional characterization is necessary to show that these neurons are inhibitory. Validation of these minor clusters would require an even larger sample of recorded layer 6 neurons.

\section{Implications of clusters}

If the functional subpopulations described in the current study are related to the structurally defined subclasses in layer 6 (Wiser and Callaway, 1996; Hasse et al., 2019), then there will be separate target regions for the axons of these neurons. The diversity of functional types may be related to the function of their target sites. Many layer 6 neurons project intracortically (Wiser and Callaway, 1996), and some have axons preferentially terminating in layer $4 \mathrm{C} \alpha$, the major M-pathway recipient sublayer in $\mathrm{V} 1$, whereas others that preferentially arborize in layer $4 \mathrm{C} \beta$ and layer $4 \mathrm{~A}$, the principal $\mathrm{P}$-pathway recipient zones of layer 4 . Although it is currently not known how these anatomical subclasses map onto our functionally defined clusters, if functional selectivity maps to anatomical classes, L6 neurons in different functional clusters could provide cluster-specific feedback. Approximately $10 \%-15 \%$ of layer 6 neurons provide feedback projections to the LGN (Fitzpatrick et al., 1994), and these have some functional attributes (Briggs and Usrey, 2009; Hasse and Briggs, 2017) that align mainly with two of the clusters (C2 and C6) we have identified. Another structurally defined group of neurons project to extrastriate visual area MT (Lund et al., 1975; Fries et al., 1985; Nhan and Callaway, 2012). Movshon and Newsome (1996) showed that some layer 6 neurons projecting to MT have functional properties similar to those of $\mathrm{C} 1$. Future studies combining functional and anatomical characterization may reveal whether these functional clusters indeed correspond to particular anatomical classes.

\section{References}

Barlow HB, Kaushal TP, Hawken M, Parker AJ (1987) Human contrast discrimination and the threshold of cortical neurons. J Opt Soc Am A 4:2366-2371.

Bonds AB (1991) Temporal dynamics of contrast gain in single cells of the cat striate cortex. Vis Neurosci 6:239-255.

Briggs F, Callaway EM (2001) Layer-specific input to distinct cell types in layer 6 of monkey primary visual cortex. J Neurosci 21:3600-3608.

Briggs F, Usrey WM (2009) Parallel processing in the corticogeniculate pathway of the macaque monkey. Neuron 62:135-146.

Briggs F, Kiley CW, Callaway EM, Usrey WM (2016) Morphological substrates for parallel streams of corticogeniculate feedback originating in both V1 and V2 of the macaque monkey. Neuron 90:388-399.

Callaway EM (1998) Local circuits in primary visual cortex of the macaque monkey. Annu Rev Neurosci 21:47-74.

Campbell F, Fiorentini A (1973) The visual cortex as a spatial frequency analyzer. Vis Res 13:1255-1267.

Chariker L, Shapley R, Young LS (2016) Orientation selectivity from very sparse LGN inputs in a comprehensive model of macaque V1 cortex. J Neurosci 36:12368-12384.

Chariker L, Shapley R, Young LS (2018) Rhythm and synchrony in a cortical network model. J Neurosci 38:8621-8634.

Chirimuuta M, Tolhurst DJ (2005a) Does a Bayesian model of V1 contrast coding offer a neurophysiological account of human contrast discrimination? Vis Res 45:2943-2959.

Chirimuuta M, Tolhurst DJ (2005b) Accuracy of identification of grating contrast by human observers: Bayesian models of V1 contrast processing show correspondence between discrimination and identification performance. Vis Res 45:2960-2971.

Constantinople CM, Disney AA, Maffie J, Rudy B, Hawken MJ (2009) Quantitative analysis of neurons with Kv3 potassium channel subunits, $\mathrm{Kv} 3.1 \mathrm{~b}$ and Kv3.2, in macaque primary visual cortex. J Comp Neurol 516:291-311.

De Valois RL, Albrecht DG, Thorell LG (1982a) Spatial frequency selectivity of cells in macaque visual cortex. Vis Res 22:545-559.

De Valois RL, Yund EW, Hepler N (1982b) The orientation and direction selectivity of cells in macaque visual cortex. Vis Res 22:531-544.

Fitzpatrick D, Lund JS, Blasdel GG (1985) Intrinsic connections of macaque striate cortex: afferent and efferent connections of lamina 4C. J Neurosci 5:3329-3349. 
Fitzpatrick D, Usrey WM, Schofield BR, Einstein G (1994) The sublaminar organization of corticogeniculate neurons in layer 6 of macaque striate cortex. Vis Neurosci 11:307-315.

Fries W, Keizer K, Kuypers HG (1985) Large layer VI cells in macaque striate cortex (Meynert cells) project to both superior colliculus and prestriate visual area V5. Exp Brain Res 58:613-616.

Garcia-Marin V, Ahmed TH, Azfal YC, Hawken MJ (2013) The distribution vesicular glutamate transporter (VGluT2) in the primary visual cortex of macaque and human. J Comp Neurol 521:130-151.

Gegenfurtner KR, Kiper DC, Beusmans JM, Carandini M, Zaidi Q, Movshon JA (1994) Chromatic properties of neurons in macaque MT. Vis Neurosci 11:455-466.

Gilbert CD (1977) Laminar differences in receptive field properties of cells in cat primary visual cortex. J Physiol 268:391-421.

Hasse JM, Briggs F (2017) A cross-species comparison of corticogeniculate structure and function. Vis Neurosci 34:E016.

Hasse JM, Bragg EM, Murphy AJ, Briggs F (2019) Morphological heterogeneity among corticogeniculate neurons in ferrets: quantification and comparison with a previous report in macaque monkeys. J Comp Neurol 527:546-557.

Hawken MJ, Parker AJ (1987) Spatial properties of neurons in the monkey striate cortex. Proc R Soc Lond B Biol Sci 231:251-288.

Hawken MJ, Parker AJ, Lund JS (1988) Contrast sensitivity and laminar organization of direction-selective cells in the striate cortex of the OldWorld monkey. J Neurosci 8:3541-3548.

Hawken MJ, Shapley RM, Grosof DH (1996) Temporal frequency selectivity in monkey lateral geniculate nucleus and striate cortex. Vis Neurosci 13:477-492.

Hendrickson AE, Wilson JR, Ogren MP (1978) The neuroanatomical organization of pathways between the dorsal lateral geniculate nucleus and visual cortex in Old World and New World primates. J Comp Neurol 182:123-136.

Henry CA, Hawken MJ (2013) Stability of simple-complex classification with contrast and extra-classical receptive field modulation in macaque V1. J Neurophysiol 109:1793-1803.

Henry CA, Joshi S, Xing D, Shapley RM, Hawken MJ (2013) Functional characterization of the extraclassical receptive field in macaque V1: contrast, orientation and temporal dynamics. J Neurosci 33:6230-6242.

Hubel DH, Wiesel TN (1962) Receptive fields, binocular interaction and functional architecture in the cat's visual cortex. J Physiol 160:106-154.

Hubel DH, Wiesel TN (1968) Receptive fields and functional architecture of monkey striate cortex. J Physiol 195:215-243.

Ichinohe N, Watakabe A, Miyashita T, Yamamori T, Hashikawa T, Rockland KS (2004) A voltage-gated potassium channel, Kv3.1b, is expressed by a subpopulation of large pyramidal neurons in layer 5 of the macaque monkey cortex. Neurosci 129:179-185.

Kelly JG, García-Marín V, Rudy B, Hawken MJ (2019) Densities and laminar distributions of Kv3.1b-, PV-, GABA-, and SMI-32-immunoreactive neurons in macaque area V1. Cereb Cortex 29:1921-1937.

Legge GE (1981) A power law for visual contrast discrimination. Vis Res 21:457-467.

Livingstone MS, Hubel DH (1984) Anatomy and physiology of a color system in the primate visual cortex. J Neurosci 4:309-356.

Lund JS, Boothe R (1975) Interlaminar connections and pyramidal neuron organization in the visual cortex, area 17, of the macaque monkey. J Comp Neurol 159:305-334.

Lund JS, Lund RD, Hendrickson AE, Bunt AH, Fuchs AF (1975) The origin of efferent pathways from the primary visual cortex, area 17 , of the macaque monkey. J Comp Neurol 164:287-303.

McGuire BA, Hornung JP, Gilbert CD, Wiesel TN (1984) Patterns of synaptic input to layer 4 of cat striate cortex. J Neurosci 4:3021-3033.

Movshon JA, Newsome WT (1996) Visual response properties of striate cortical neurons projecting to area MT in macaque monkeys. J Neurosci 16:7733-7741.

Movshon JA, Thompson ID, Tolhurst DJ (1978) Spatial summation in the receptive fields of simple cells in the cat's striate cortex. J Physiol 283:53-77.

Nhan HL, Callaway EM (2012) Morphology of superior-colliculus- and middle temporal area-projecting neurons in primate primary visual cortex. J Comp Neurol 520:52-80.

Nowak LG, Sanchez-Vives MV, McCormick DA (2008) Lack of orientation and direction selectivity in a subgroup of fast-spiking inhibitory interneurons: cellular and synaptic mechanisms and comparison with other electrophysiological cell types. Cereb Cortex 18:1058-1078.

Orban GA, Kennedy H, Bullier J (1986) Velocity sensitivity and direction selectivity of neurons in areas V1 and V2 of the monkey: influence of eccentricity. J Neurophysiol 56:462-480.

Parker AJ, Hawken MJ (1988) Two-dimensional spatial structure of receptive fields in the monkey striate cortex. J Opt Soc Am A 5:598-605.

Peirce JW (2007) The potential importance of saturating and supersaturating contrast response functions in visual cortex. J Vis 7:13.

Ringach DL, Shapley RM, Hawken MJ (2002) Orientation selectivity in macaque V1: diversity and laminar dependence. J Neurosci 22:5639-5651.

Rudy B, McBain CJ (2001) Kv3 channels: voltage-gated $\mathrm{K}^{+}$channels designed for high-frequency repetitive firing. Trends Neurosci 24:517-526.

Sceniak MP, Ringach DL, Hawken MJ, Shapley R (1999) Contrast's effect on spatial summation by V1 neurons. Nat Neurosci 2:733-739.

Sceniak MP, Hawken MJ, Shapley R (2001) Visual spatial characterization of macaque V1 neurons. J Neurophysiol 85:1873-1887.

Schiller PH, Finlay BL, Volman SF (1976a) Quantitative studies of singlecell properties in monkey striate cortex: I. Spatiotemporal organization of receptive fields. J Neurophysiol 39:1288-1319.

Schiller PH, Finlay BL, Volman SF (1976b) Quantitative studies of singlecell properties in monkey striate cortex: V. Multivariate statistical analyses and models. J Neurophysiol 39:1362-1374.

Skottun BC, De Valois RL, Grosof DH, Movshon JA, Albrecht DG, Bonds AB (1991) Classifying simple and complex cells on the basis of response modulation. Vis Res 31:1079-1086.

Solomon SG, Lee BB, Sun H (2006) Suppressive surrounds and contrast gain in magnocellular-pathway retinal ganglion cells of macaque. J Neurosci 26:8715-8726.

Tolhurst DJ, Thompson ID (1981) On the variety of spatial frequency selectivities shown by neurons in area 17 of the cat. Proc R Soc Lond B Biol Sci 213:183-199.

Wiser AK, Callaway EM (1996) Contributions of individual layer 6 pyramidal neurons to local circuitry in macaque primary visual cortex. J Neurosci 16:2724-2739.

Xing D, Shapley RM, Hawken MJ. Ringach DL (2005) The effect of stimulus size on the dynamics of orientation selectivity in macaque V1. J Neurophysiol 94:799-812.

Zeki SM (1974) Functional organization of a visual area in the posterior bank of the superior temporal sulcus of the rhesus monkey. J Physiol 236:549-573 